\title{
Coinduction Up-To in a Fibrational Setting *
}

\author{
Filippo Bonchi Daniela Petrişan \\ Damien Pous ${ }^{\dagger}$ \\ LIP, CNRS, INRIA, ENS Lyon, \\ Université de Lyon, UMR 5668 \\ \{filippo.bonchi,daniela.petrisan,damien. pous\} \\ Qens-lyon.fr
}

\author{
Jurriaan Rot ${ }^{\ddagger}$ \\ LIACS - Leiden University, CWI \\ j.c.rot@liacs.leidenuniv.nl
}

\begin{abstract}
Bisimulation up-to enhances the coinductive proof method for bisimilarity, providing efficient proof techniques for checking properties of different kinds of systems. We prove the soundness of such techniques in a fibrational setting, building on the seminal work of Hermida and Jacobs. This allows us to systematically obtain up-to techniques not only for bisimilarity but for a large class of coinductive predicates modelled as coalgebras. By tuning the parameters of our framework, we obtain novel techniques for unary predicates and nominal automata, a variant of the GSOS rule format for similarity, and a new categorical treatment of weak bisimilarity.
\end{abstract}

Categories and Subject Descriptors F.3 [Logics and meanings of programs]; F.4 [Mathematical logic and formal languages]

General Terms Theory.

Keywords fibrations, coinductive predicates, bisimulation up-to, GSOS, up-to techniques, similarity, bialgebras, nominal automata.

\section{Introduction}

\subsection{Coinduction up-to}

The rationale behind coinductive up-to techniques is the following. Suppose you have a characterisation of an object of interest as a greatest fixed-point. For instance, behavioural equivalence in CCS is the greatest fixed-point of a monotone function $B$ on relations, describing the standard bisimulation game. This means that to prove two processes equivalent, it suffices to exhibit a relation $R$ that relates them, and which is a $B$-invariant, i.e., $R \subseteq B(R)$. Such a task can however be painful or inefficient, and one could prefer to exhibit a relation which is only a $B$-invariant up to some function $A$, i.e., $R \subseteq B(A(R))$.

Not every function $A$ can safely be used: $A$ should be sound for $B$, meaning that any $B$-invariant up to $A$ should be contained in a $B$-invariant. Instances of sound functions for behavioural equivalence in process calculi usually include transitive closure, context closure and congruence closure. The use of such techniques dates back to Milner's work on CCS [21]; a famous example of an unsound technique is that of weak bisimulation up to weak bisimilarity. Since then, coinduction up-to proved useful, if not essential,

\footnotetext{
* Extended version of the paper with the same title, to appear in Proc. CSLLICS 2014, July 14-18, 2014, Vienna, Austria, and to be available at http://dx.doi.org/10.1145/2603088.2603149

$\dagger$ The first three authors acknowledge support from the ANR projects 2010BLAN-0305 PiCoq and 12IS02001 PACE.

$¥$ The research of this author has been funded by the Netherlands Organisation for Scientific Research (NWO), CoRE project, dossier number: 612.063 .920 .
}

in numerous proofs about concurrent systems (see [25] for a list of references); it has been used to obtain decidability results [8], and more recently to improve standard automata algorithms [7].

The theory underlying these techniques was first developed by Sangiorgi [27]. It was then reworked and generalised by one of the authors to the abstract setting of complete lattices [24, 25]. The key observation there is that the notion of soundness is not compositional: the composition of two sound functions is not necessarily sound itself. The main solution to this problem consists in restricting to compatible functions, a subset of the sound functions which enjoys nice compositionality properties and contains most of the useful techniques.

An illustrative example of the benefits of a modular theory is the following: given a signature $\Sigma$, consider the congruence closure function, that is, the function $C g r$ mapping a relation $R$ to the smallest congruence containing $R$. This function has proved to be useful as an up-to technique for language equivalence of non-deterministic automata [7]. It can be decomposed into small pieces as follows: $C g r=\operatorname{Trn} \circ S y m \circ C t x \circ R f l$, where Trn is the transitive closure, Sym is the symmetric closure, $R f$ is the reflexive closure, and Ctx is the context closure associated to $\Sigma$. Since compatibility is preserved by composition (among other operations), the compatibility of $\mathrm{Cgr}$ follows from that of its smaller components. In turn, transitive closure can be decomposed in terms of relational composition, and context closure can be decomposed in terms of the smaller functions that close a relation with respect to $\Sigma$ one symbol at a time. Compatibility of such functions can thus be obtained in a modular way.

A key observation in the present work is that when we move to a coalgebraic presentation of the theory, compatible functions generalise to functors equipped with a distributive law (Section 3).

\subsection{Fibrations and coinductive predicates}

Coalgebras are a tool of choice for describing state based systems: given a functor $F$ determining its type (e.g., labelled transition systems, automata, streams), a system is just an $F$-coalgebra $(X, \xi)$. When $F$ has a final coalgebra $(\Omega, \omega)$, this gives a canonical notion of behavioural equivalence [17]:

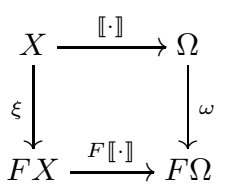

two states $x, y \in X$ are equivalent if they are mapped to the same element in the final coalgebra.

When the functor $F$ preserves weak pullbacks-which we shall assume throughout this introductory section for the sake of 
simplicity-behavioural equivalence can be characterised coinductively using Hermida-Jacobs bisimulations [14, 30]: given an $F$-coalgebra $(X, \xi)$, behavioural equivalence is the largest $B$ invariant for a monotone function $B$ on $\operatorname{Rel}_{X}$, the poset of binary relations over $X$. This function $B$ can be decomposed as

$$
B \triangleq \xi^{*} \circ \operatorname{Rel}(F)_{X}: \operatorname{Rel}_{X} \rightarrow \operatorname{Rel}_{X}
$$

Let us explain the notations used here. We consider the category Rel whose objects are relations $R \subseteq X^{2}$ and morphisms from $R \subseteq X^{2}$ to $S \subseteq Y^{2}$ are maps from $X$ to $Y$ sending pairs in $R$ to pairs in $S$. For each set $X$ the poset $\operatorname{Rel}_{X}$ of binary relations over $X$ is a subcategory of Rel, also called the fibre over $X$. The functor $F$ has a canonical lifting to $\operatorname{Rel}$, denoted by $\operatorname{Rel}(F)$. This lifting restricts to a functor $\operatorname{Rel}(F)_{X}: \operatorname{Rel}_{X} \rightarrow \operatorname{Rel}_{F X}$, which in this case is just a monotone function between posets. The monotone function $\xi^{*}: \operatorname{Rel}_{F X} \rightarrow \operatorname{Rel}_{X}$ is the inverse image of the coalgebra $\xi$ mapping a relation $R \subseteq(F X)^{2}$ to $(\xi \times \xi)^{-1}(R)$.

To express other predicates than behavioural equivalence, one can take arbitrary liftings of $F$ to Rel, different from the canonical one. Any lifting $\bar{F}$ yields a functor $B$ defined as

$$
B \triangleq \xi^{*} \circ \bar{F}_{X}: \operatorname{Rel}_{X} \rightarrow \operatorname{Rel}_{X}
$$

The final coalgebra, or greatest fixed-point for such a $B$ is called a coinductive predicate $[13,14]$. By taking appropriate $\bar{F}$, one can obtain, for instance, various behavioural preorders: similarity on labelled transition systems (LTSs), language inclusion on automata, or lexicographic ordering of streams.

This situation can be further generalised using fibrations. We refer the reader to the first chapter of [16] for a gentle introduction, or to Section 2 for succinct definitions. The functor $p: \operatorname{Rel} \rightarrow$ Set mapping a relation $R \subseteq X^{2}$ to its support set $X$ is a fibration, where the inverse image $\xi^{*}$ is just the reindexing functor of $\xi$. By choosing a different fibration than Rel, one can obtain coinductive characterisations of objects that are not necessarily binary relations, e.g., unary predicates like divergence, ternary relations, or metrics.

Our categorical generalisation of compatible functions provides a natural extension of this fibrational framework with a systematic treatment of up-to techniques: we provide functors (i.e., monotone functions in the special case of the Rel fibration) that are compatible with those functors $B$ corresponding to coinductive predicates.

For instance, when the chosen lifting $\bar{F}$ is a fibration map, the functor corresponding to a technique called "up to behavioural equivalence" is compatible (Theorem 1). The canonical lifting of a functor is always such a fibration map, so that when $F$ is the functor for LTSs, we recover the soundness of the very first up-to technique from the literature, namely "bisimulation up to bisimilarity" [21]. One can also check that another lifting of this same functor but in another fibration yields the divergence predicate, and is a fibration map. We thus obtain the validity of the "divergence up to bisimilarity" technique.

\subsection{Bialgebras and up to context}

Another important class of techniques comes into play when considering systems with an algebraic structure on the state space (e.g., the syntax of a process calculus). A minimal requirement for such systems usually is that behavioural equivalence should be a congruence. In the special case of bisimilarity on LTSs, several rule formats have been proposed to ensure such a congruence property [1]. At the categorical level, the main concept to study such systems is that of bialgebras. Assume two endofunctors $T, F$ related by a distributive law $\lambda: T F \Rightarrow F T$. A $\lambda$-bialgebra consists in a triple $(X, \alpha, \xi)$ where $(X, \alpha)$ is a $T$-algebra, $(X, \xi)$ is an $F$-coalgebra, and a diagram involving $\lambda$ commutes. It is well known that in such a bialgebra, behavioural equivalence is a congruence with respect to $T$ [31]. This is actually a generalisation of the fact that bisimi- larity is a congruence for all GSOS specifications [3]: GSOS specifications are in one-to-one correspondence with distributive laws between the appropriate functors $[2,31]$.

This congruence result can be strengthened into a compatibility result [26]: in any $\lambda$-bialgebra, the contextual closure function that corresponds to $T$ is compatible for behavioural equivalence. By moving to fibrations, we generalise this result so that we can obtain up to context techniques for arbitrary coinductive predicates: unary predicates like divergence, by using another fibration than Rel; but also other relations than behavioural equivalence, like the behavioural preorders mentioned above, or weak bisimilarity.

The technical device we need to establish this result is that of bifibrations, fibrations $p$ whose opposite functor $p^{o p}$ is also a fibration. We keep the running example of the Rel fibration for the sake of clarity; the results are presented in full generality in the remaining parts of the paper. In such a setting, any morphism $f: X \rightarrow Y$ in Set has a direct image $\coprod_{f}: \operatorname{Rel}_{X} \rightarrow \operatorname{Rel}_{Y}$. Now given an algebra $\alpha: T X \rightarrow X$ for a functor $T$ on Set, any lifting $\bar{T}$ of $T$ gives rise to a functor on the fibre above $X$, defined dually to $(\dagger)$ :

$$
C \triangleq \coprod_{\alpha} \circ \bar{T}_{X}: \operatorname{Rel}_{X} \rightarrow \operatorname{Rel}_{X}
$$

When we take for $\bar{T}$ the canonical lifting of $T$ in $\operatorname{Rel}$, then $C$ is the contextual closure function corresponding to the functor $T$. We shall see that we sometimes need to consider variations of the canonical lifting to obtain a compatible up-to technique (e.g., up to "monotone" contexts for checking language inclusion of weighted automata-Section 5.1).

Now, starting from a $\lambda$-bialgebra $(X, \alpha, \xi)$, and given two liftings $\bar{T}$ and $\bar{F}$ of $T$ and $F$, respectively, the question is whether the above functor $C$ is compatible with the functor $B$ defined earlier in $(\dagger)$. The simple condition we give in this paper is the following: the distributive law $\lambda: T F \Rightarrow F T$ should lift to a distributive law $\bar{\lambda}: \bar{T} \bar{F} \Rightarrow \bar{F} \bar{T}$ (Theorem 2).

This condition is always satisfied in the bifibration Rel, when $\bar{T}$ and $\bar{F}$ are the canonical liftings of $T$ and $F$. Thus we obtain as a corollary the compatibility of bisimulation of up to context in $\lambda$-bialgebras, which is the main result from [26]—-soundness was previously observed by Lenisa et al. [19, 20] and then Bartels [2].

The present work allows us to go further in several directions, as illustrated below.

\subsection{Contributions and Applications}

The main contribution of this paper is the abstract framework developed in Section 4; it allows us to derive the soundness of a wide range of both novel and well-established up-to techniques for arbitrary coinductive predicates. Sections 5 and 6 are devoted to several such applications, which we describe now.

When working in the predicate fibration on Set, one can characterise some formulas from modal logic as coinductive predicates (see [9] for an account of coalgebraic modal logic). Our framework allows us to introduce up-to techniques in this setting: we consider the formula $\nu x .\langle\tau\rangle x$ in Section 5.2, and we provide a technique called "divergence up to left contexts and behavioural equivalence". We use it to prove divergence of a simple process using a finite invariant, while the standard method requires an infinite one.

One can also change the base category: by considering the fibration of equivariant relations over nominal sets, we show how to obtain up-to techniques for language equivalence of non-deterministic nominal automata [4]. In Section 5.3, these techniques allow us to prove the equivalence of two nominal automata using an orbit-finite relation, where the standard method would require an infinite one (recall that the determinisation of a nominal automaton is not necessarily orbit-finite). 
Another benefit of the presented theory is modularity w.r.t. the liftings chosen to define coinductive predicates: two liftings can be composed, and we give sufficient conditions for deriving compatible functors for the composite lifting out of compatible functors for its sub-components (Section 6). We give two examples of such a situation: similarity, and weak bisimilarity on LTSs.

By using Hughes and Jacobs' definition of similarity [15], we obtain that for "up to context" to be compatible it suffices to start from a monotone distributive law (Section 6.1). In the special case of LTSs, this monotonicity condition amounts to the positive GSOS rule format [12]: GSOS [3] without negative premises.

In Section 6.2 we propose a novel characterisation of weak bisimilarity on LTSs, that fits into our framework. This allows us to give a generic condition for "up to context" to be compatible (and hence weak bisimilarity to be a congruence). In particular, this condition rules out the sum operation from CCS, which is well known not to preserve weak bisimilarity.

\section{Preliminaries}

We refer the reader to [16] for background on fibrations and recall here basic definitions.

Definition 1. A functor $p: \mathcal{E} \rightarrow \mathcal{B}$ is called a fibration when for every morphism $f: X \rightarrow Y$ in $\mathcal{B}$ and every $R$ in $\mathcal{E}$ with $p(R)=Y$ there exists a map $\widetilde{f_{R}}: f^{*}(R) \rightarrow R$ such that $p\left(\widetilde{f_{R}}\right)=f$ satisfying the universal property: For all maps $g: Z \rightarrow X$ in $\mathcal{B}$ and $u: Q \rightarrow R$ in $\mathcal{E}$ sitting above $f g$ (i.e., $p(u)=f g$ ) there is a unique map $v: Q \rightarrow f^{*}(R)$ such that $u=\widetilde{f_{R}} v$ and $p(v)=g$.
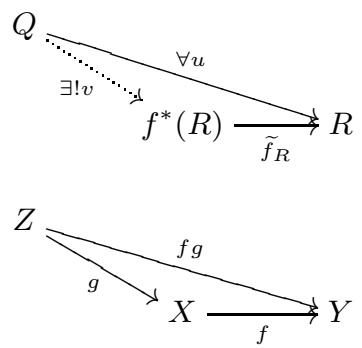

For $X$ in $\mathcal{B}$ we denote by $\mathcal{E}_{X}$ the fibre above $X$, i.e., the subcategory of $\mathcal{E}$ with objects mapped by $p$ to $X$ and arrows sitting above the identity on $X$.

A map $\widetilde{f}$ as above is called a Cartesian lifting of $f$ and is unique up to isomorphism. If we make a choice of Cartesian liftings, the association $R \mapsto f^{*}(R)$ gives rise to the so-called reindexing functor $f^{*}: \mathcal{E}_{Y} \rightarrow \mathcal{E}_{X}$.

The fibrations considered in this paper are bicartesian (both $\mathcal{E}$ and $\mathcal{B}$ have a bicartesian structure strictly preserved by $p$ ) and split, i.e., the reindexing functors behave well with respect to composition and identities: $\left(1_{X}\right)^{*}=1_{\mathcal{E}_{X}}$ and $(f \circ g)^{*}=g^{*} \circ f^{*}$.

A functor $p: \mathcal{E} \rightarrow \mathcal{B}$ is called a bifibration if both $p: \mathcal{E} \rightarrow \mathcal{B}$ and $p^{o p}: \mathcal{E}^{o p} \rightarrow \mathcal{B}^{o p}$ are fibrations. A fibration $p: \mathcal{E} \rightarrow \mathcal{B}$ is a bifibration if and only if each reindexing functor $f^{*}: \mathcal{E}_{Y} \rightarrow \mathcal{E}_{X}$ has a left adjoint $\coprod_{f} \dashv f^{*}$, see [16, Lemma 9.1.2].

Example 1. Let Pred be the category of predicates: objects are pairs of sets $(P, X)$ with $P \subseteq X$ and morphisms $f:(P, X) \rightarrow$ $(Q, Y)$ are arrows $f: X \rightarrow Y$ that can be restricted to $\left.f\right|_{P}: P \rightarrow$ $Q$.

Similarly, we can consider the category Rel whose objects are pairs of sets $(R, X)$ with $R \subseteq X^{2}$ and morphisms $f:(R, X) \rightarrow$ $(S, Y)$ are arrows $f: X \rightarrow Y$ such that $f \times f$ can be restricted to $f \times\left. f\right|_{R}: R \rightarrow S$.

The functors mapping predicates, respectively, relations to their underlying sets are bifibrations. The fibres $\operatorname{Pred}_{X}$ and $\operatorname{Rel}_{X}$ sitting above $X$ are the posets of subsets of $X$, respectively relations on $X$, ordered by inclusion. The reindexing functors are given by inverse image and their left adjoints by direct image.

Given fibrations $p: \mathcal{E} \rightarrow \mathcal{B}$ and $p^{\prime}: \mathcal{E}^{\prime} \rightarrow \mathcal{B}$ and $F: \mathcal{B} \rightarrow \mathcal{B}$, we call $\bar{F}: \mathcal{E} \rightarrow \mathcal{E}^{\prime}$ a lifting of $F$ when $p^{\prime} \bar{F}=F p$. Notice that a lifting $\bar{F}$ restricts to a functor between the fibres $\bar{F}_{X}: \mathcal{E}_{X} \rightarrow \mathcal{E}_{F X}$. When the subscript $X$ is clear from the context we will omit it.

A fibration map between $p: \mathcal{E} \rightarrow \mathcal{B}$ and $p^{\prime}: \mathcal{E}^{\prime} \rightarrow \mathcal{B}$ is a pair $(\bar{F}, F)$ such that $\bar{F}$ is a lifting of $F$ that preserves the Cartesian liftings: $(F f)^{*} \bar{F}=\bar{F} f^{*}$ for any $\mathcal{B}$-morphism $f$. We denote by $\operatorname{Fib}(\mathcal{B})$ the category of fibrations with base $\mathcal{B}$.

Example 2. A Set-endofunctor $T$ has a canonical relation lifting $\operatorname{Rel}(T): \operatorname{Rel} \rightarrow \operatorname{Rel}$. Represent $R \in \operatorname{Rel}_{X}$ as a jointly mono span $X \leftarrow R \rightarrow X$ and apply $T$. Then $\operatorname{Rel}(T)(R)$ is obtained by factorising the induced map $T R \rightarrow T X \times T X$. When $T$ preserves weak pullbacks, $(\operatorname{Rel}(T), T)$ is a fibration map (see e.g. [15]).

\section{Compatible Functors}

Given two monotone functions $A, B: \mathcal{C} \rightarrow \mathcal{C}$ on a complete lattice $\mathcal{C}, A$ is said to be $B$-compatible if $A B \subseteq B A$. In [25, Theorem 6.3.9], it is shown that any $B$-compatible function $A$ is sound, that is, it can be used as an up-to technique: every $B$-invariant up to $A$ is included in a $B$-invariant.

This result is an instance of a more general fact which holds in any category $\mathcal{C}$ with countable coproducts and for any pair of endofunctors $A, B$ equipped with a distributive law $\gamma: A B \Rightarrow$ $B A$. Indeed, following the proof of [2, Theorem 3.8], for any $B A$-coalgebra $\xi$ (that is a $B$-invariant up to $A$ ) one can find a $B$-coalgebra $\zeta$ (that is a $B$-invariant) making the next diagram commutative.

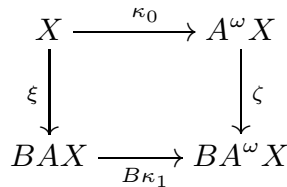

(Here $A^{\omega}$ denotes the coproduct $\coprod_{i<\omega} A^{i}$ of all finite iterations of $A$ and $\kappa_{0}, \kappa_{1}$ are the injections of $X$ and $A X$ respectively, into $A^{\omega} X$. Alternatively, we can replace the countable coproduct $A^{\omega}$ by the free monad on $A$, assuming the latter exists. In this case, the result is an instance of the generalized powerset construction [28].)

Similarly, that compatible functions preserve bisimilarity [25, Lemma 6.4.3] is an instance of the well-known fact [31] that a final $B$-coalgebra $\nu B$ lifts to a final $\gamma$-bialgebra for $\gamma: A B \Rightarrow B A$. When $\mathcal{C}$ is a lattice, this entails that $A(\nu B) \subseteq \nu B$. For instance, if $B$ is a predicate for bisimilarity and $A$ is the congruence closure function, we obtain that bisimilarity is a congruence whenever the congruence closure function is compatible.

As discussed in the Introduction, the main interest in compatible functions comes from their nice compositionality properties. This leads us to define compatibility of arbitrary functors of type $\mathcal{C} \rightarrow \mathcal{C}^{\prime}$ rather than just endofunctors.

Definition 2. Consider two endofunctors $B: \mathcal{C} \rightarrow \mathcal{C}$ and $B^{\prime}:$ $\mathcal{C}^{\prime} \rightarrow \mathcal{C}^{\prime}$. We say that a functor $A: \mathcal{C} \rightarrow \mathcal{C}^{\prime}$ is $\left(B, B^{\prime}\right)$-compatible when there exists a natural transformation $\gamma: A B \Rightarrow B^{\prime} A$.

Notice that the pair $(A, \gamma)$ is a morphism between endofunctors $B$ and $B^{\prime}$ in the sense of [20]. Since the examples dealt with in this paper involve only poset fibrations, we will omit the natural transformation $\gamma$ from the notation. Moreover, given an endofunctor $B: \mathcal{C} \rightarrow \mathcal{C}$, we will simply write that $A: \mathcal{C}^{n} \rightarrow \mathcal{C}^{m}$ is $B$-compatible, when $A$ is $\left(B^{n}, B^{m}\right)$-compatible.

This definition makes it possible to use the internal notions of product and pairing to emphasise the compositionality aspect. For 
instance, coproduct becomes a compatible functor by itself, rather than a way to compose compatible functors.

Proposition 1. Compatible functors are closed under the following constructions:

(i) composition: if $A$ is $(B, C)$-compatible and $A^{\prime}$ is $(C, D)$ compatible, then $A^{\prime} \circ A$ is $(B, D)$-compatible;

(ii) pairing: if $\left(A_{i}\right)_{i \in \iota}$ are $(B, C)$-compatible, then $\left\langle A_{i}\right\rangle_{i \in \iota}$ is $\left(B, C^{\iota}\right)$-compatible;

(iii) product: if $A$ is $(B, C)$-compatible and $A^{\prime}$ is $\left(B^{\prime}, C^{\prime}\right)$ compatible, then $A \times A^{\prime}$ is $\left(B \times B^{\prime}, C \times C^{\prime}\right)$-compatible;

Moreover, for an endofunctor $B: \mathcal{C} \rightarrow \mathcal{C}$,

(iv) the identity functor $I d: \mathcal{C} \rightarrow \mathcal{C}$ is $B$-compatible;

(v) the constant functor to the carrier of any B-coalgebra is Bcompatible, in particular the final one if it exists;

(vi) the coproduct functor $\amalg: \mathcal{C}^{\iota} \rightarrow \mathcal{C}$ is $\left(B^{\iota}, B\right)$-compatible.

\section{Up-to Techniques in a Fibration}

Throughout this section we fix a bifibration $p: \mathcal{E} \rightarrow \mathcal{B}$, an endofunctor $F: \mathcal{B} \rightarrow \mathcal{B}$, a lifting $\bar{F}: \mathcal{E} \rightarrow \mathcal{E}$ of $F$ and a coalgebra $\xi: X \rightarrow F X$. Intuitively, the studied system lives in the base category $\mathcal{B}$ while its properties live in $\mathcal{E}_{X}$, the fibre above $X$. We thus instantiate the category $\mathcal{C}$ from the previous section with $\mathcal{E}_{X}$.

As explained in the Introduction $(\dagger)$, we discuss proof techniques for the properties modelled as final coalgebras of the functor $\xi^{*} \circ \bar{F}_{X}: \mathcal{E}_{X} \rightarrow \mathcal{E}_{X}$, that we refer hereafter as $\bar{F}_{\xi}$. In Rel, when $\bar{F}$ is the canonical lifting, $\operatorname{Rel}(F)_{\xi \text {-coalgebras are exactly }}$ the Hermida-Jacobs bisimulations [14].

To obtain sound techniques for $\bar{F}_{\xi}$, it suffices to find $\bar{F}_{\xi^{-}}$ compatible endofunctors on $\mathcal{E}_{X}$. We provide such functors by giving conditions on the lifting $\bar{F}$, abstracting away from the coalgebra $\xi$ at hand.

\subsection{Compatibility of Behavioural Equivalence Closure}

The most basic technique is up to behavioural equivalence, a prime example of which is Milner's up to bisimilarity [21], where a relation $R$ is mapped into $\sim R \sim$. If $f$ is the unique morphism from $\xi$ to a final $F$-coalgebra (assumed to exist), behavioural equivalence is the kernel of $f$. This leads us to consider the functor

$$
B h v=f^{*} \circ \coprod_{f}: \mathcal{E}_{X} \rightarrow \mathcal{E}_{X} \text {. }
$$

For the fibrations Pred $\rightarrow$ Set and Rel $\rightarrow$ Set the functor $B h v$ maps a predicate, respectively a relation, to its closure under behavioural equivalence. The compatibility of $B h v$ is an instance of:

Theorem 1. Suppose that $(\bar{F}, F)$ is a fibration map. For any $F$ coalgebra morphism $f:(X, \xi) \rightarrow(Y, \zeta)$, the functor $f^{*} \circ \coprod_{f}$ is $\bar{F}_{\xi}$-compatible.

Proof sketch. We exhibit a natural transformation

$$
f^{*} \circ \coprod_{f} \circ\left(\xi^{*} \circ \bar{F}\right) \Rightarrow\left(\xi^{*} \circ \bar{F}\right) \circ f^{*} \circ \coprod_{f}
$$

obtained by pasting the 2-cells $(a),(b),(c),(d)$ in the following diagram:

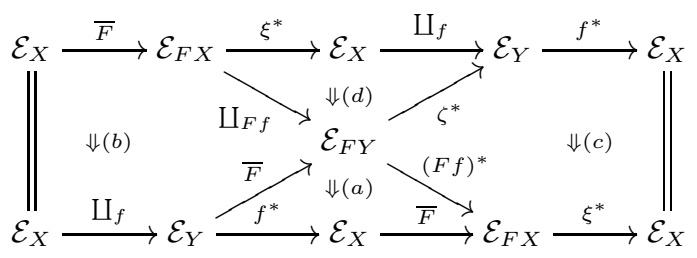

(a) Since $(\bar{F}, F)$ is a fibration map we have that

$$
\bar{F} f^{*}=(F f)^{*} \bar{F}
$$

(b) is a consequence of Lemma 3 in Appendix B.

(c) is a natural isomorphism and comes from the fact that $f$ is a coalgebra map and the fibration is split.

(d) is obtained from $(c)$ using the counit of $\coprod_{f} \dashv f^{*}$ and the unit of $\coprod_{F f} \dashv(F f)^{*}$.

(Note that this proof decomposes into a proof that $\coprod_{f}$ is $\left(\bar{F}_{\xi}, \bar{F}_{\zeta}\right)$ compatible, by pasting (b) and (d), and a proof that $f^{*}$ is $\left(\bar{F}_{\zeta}, \bar{F}_{\xi}\right)$ compatible, by pasting (a) and (c). These two independent results can be composed by Proposition 1(i) to obtain the theorem.)

Corollary 1. If $F$ is a Set-functor preserving weak pullbacks then the behavioural equivalence closure functor Bhv is $\operatorname{Rel}(F)_{\xi}$ compatible.

Proof. $(\operatorname{Rel}(F), F)$ is a fibration map whenever $F$ preserves weak pullbacks (see e.g. [15]).

From Theorem 1 we also derive the soundness of up-to Bhv for unary predicates: the monotone predicate liftings used in coalgebraic modal logic [9] are fibration maps [17], thus the hypothesis of Theorem 1 are satisfied.

\subsection{Compatibility of Equivalence Closure}

In this section we show that compatibility of equivalence closure can be modularly derived from compatibility of reflexive, symmetric and transitive closures. For the latter it suffices to prove that relational composition is compatible. Composition of relations can be expressed in a fibrational setting, by considering the category Rel $\times$ Set Rel obtained as a pullback of the fibration Rel $\rightarrow$ Set along itself:

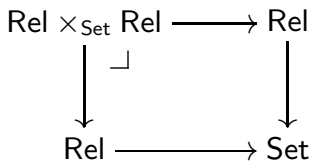

Then relational composition is a functor $\otimes: \operatorname{Rel} \times$ Set $\operatorname{Rel} \rightarrow$ Rel mapping $R, S \subseteq X \times X$ to their composition. As we will see as a corollary of Proposition 2, when proving compatibility of relational composition with respect to $\bar{F}_{\xi}$ we can abstract away from the coalgebra $\xi$ and simply use that $\otimes$ is a morphism of endofunctors from $\bar{F}^{2}$ to $\bar{F}$.

Compatibility of symmetric and reflexive closures can be proved following the same principle. This leads us to consider for an arbitrary fibration $\mathcal{E} \rightarrow \mathcal{B}$ its $n$-fold product in the category $\mathrm{Fib}(\mathcal{B})$, denoted by $\mathcal{E}^{\times \mathcal{B}^{n}} \rightarrow \mathcal{B}$. The objects in $\mathcal{E}^{\times \mathcal{B}^{n}}$ are tuples of objects in $\mathcal{E}$ belonging to the same fibre. This product is computed fibrewise, that is, $\mathcal{E}_{X}^{\times \mathcal{B} n}=\mathcal{E}_{X}^{n}$. For $n=0$ we have $\mathcal{E}^{0}=\mathcal{B}$.

Hereafter, we are interested in functors $G: \mathcal{E}^{\times \mathcal{B}^{n}} \rightarrow \mathcal{E}$ that are liftings of the identity functor on $\mathcal{B}$ : for each $X$ in $\mathcal{B}$ we have functors $G_{X}: \mathcal{E}_{X}^{n} \rightarrow \mathcal{E}_{X}$. Then relational composition is just an instance of $G$ for $n=2$.

Proposition 2. Let $G: \mathcal{E}^{\times{ }_{\mathcal{B}} n} \rightarrow \mathcal{E}$ be a lifting of the identity, with a natural transformation $G \bar{F}^{n} \Rightarrow \bar{F} G$. Then $G_{X}$ is $\bar{F}_{\xi^{-}}$. compatible.

We list now several applications of the proposition for the fibration Rel $\rightarrow$ Set.

$(n=0)$ Let $R f l$ : Set $\rightarrow$ Rel be the functor mapping each set $X$ to $\Delta_{X}$, the identity relation on $X . R f l_{X}$ is $\bar{F}_{\xi}$-compatible if

$$
\Delta_{F X} \subseteq \bar{F} \Delta_{X} \text {. }
$$


$(n=1)$ Let $S y m:$ Rel $\rightarrow$ Rel be the functor mapping each relation $R \subseteq X^{2}$ to its converse $R^{-1} \subseteq X^{2} . S y m_{X}$ is $\bar{F}_{\xi \text {-compatible }}$ if for all relations $R \subseteq X^{2}$

$$
\bar{F}(R)^{-1} \subseteq \bar{F}\left(R^{-1}\right) .
$$

$(n=2)$ Let $\otimes:$ Rel $\times_{\text {Set }} \operatorname{Rel} \rightarrow$ Rel be the relational composition functor. Then $\otimes_{X}$ is $\bar{F}_{\xi}$-compatible if for all $R, S \subseteq X^{2}$

$$
\bar{F} R \otimes \bar{F} S \subseteq \bar{F}(R \otimes S)
$$

If moreover $T_{1}, T_{2}: \operatorname{Rel}_{X} \rightarrow \operatorname{Rel}_{X}$ are two $\bar{F}_{\xi}$-compatible functors, their pointwise composition $T_{1} \otimes T_{2}=\otimes_{X} \circ\left\langle T_{1}, T_{2}\right\rangle$ is $\bar{F}_{\xi}$-compatible by Proposition 1 (i,ii).

The transitive closure functor $\operatorname{Tr} n$ is obtained from $\otimes$ in a modular way:

$$
\operatorname{Trn}=\coprod_{i \geq 0}(-)^{i}: \mathcal{E}_{X} \rightarrow \mathcal{E}_{X}
$$

where $(-)^{0}=\operatorname{Id}$ and $(-)^{i+1}=\operatorname{Id} \otimes(-)^{i}$. Using Proposition 1 we get

Corollary 2. If $F$ is a Set-functor then the reflexive and symmetric closure functors $R f_{X}$ and $S y m_{X}$ are $\operatorname{Rel}(F)_{\xi}$-compatible. Moreover, if $F$ preserves weak pullbacks, then the transitive closure functor $\operatorname{Trn}_{X}$ is $\operatorname{Rel}(F)_{\xi}$-compatible.

Proof. The above conditions $(*)$ and $(* *)$ always hold for the canonical lifting $\bar{F}=\operatorname{Rel}(F) ;(* * *)$ holds for $\operatorname{Rel}(F)$ when $F$ preserves weak pullbacks.

By compositionality (Proposition 1), one can then deduce compatibility of the equivalence closure functor: this functor can be defined as $E q v \triangleq T r n \circ(\mathrm{Id}+S y m+R f l)$, where + denotes binary coproduct.

When $\bar{F}_{\xi}$ has a final coalgebra $S$, one can define a "self closure" $\mathcal{E}_{X}$-endofunctor $S l f=\widetilde{S} \otimes \operatorname{Id} \otimes \widetilde{S}$, where $\widetilde{S}: \mathcal{E}_{X} \rightarrow \mathcal{E}_{X}$ is the constant to $S$ functor. Thanks to Proposition 1, the functor Slf

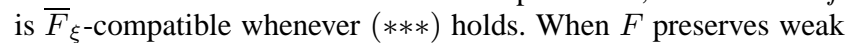
pullbacks and $\bar{F}$ is instantiated to the canonical lifting $\operatorname{Rel}(F)$, Slf coincides with $B h v$ since $S$ is just behavioural equivalence in this case. If instead we consider the lifting that yields weak bisimilarity (to be defined in Section 6.2), Slf corresponds to a technique called "weak bisimulation up to weak bisimilarity", while Bhv corresponds to "weak bisimulation up to (strong) bisimilarity".

\subsection{Compatibility of Contextual Closure}

For defining contextual closure, we assume that the state space of the coalgebra is equipped with an algebraic structure. More precisely, we fix a bialgebra for a distributive law $\lambda: T F \Rightarrow F T$, that is, a triple $(X, \alpha, \xi)$, where $\alpha: T X \rightarrow X$ is a an algebra and $\xi: X \rightarrow F X$ is a coalgebra such that the next diagram commutes:

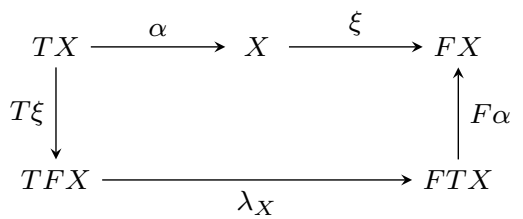

Theorem 2. Let $\bar{T}, \bar{F}: \mathcal{E} \rightarrow \mathcal{E}$ be liftings of $T$ and $F$. If $\bar{\lambda}: \bar{T} \bar{F} \Rightarrow$ $\bar{F} \bar{T}$ is a natural transformation sitting above $\lambda$, then $\coprod_{\alpha} \circ \bar{T}$ is $\bar{F}_{\xi \text {-compatible. }}$

Proof sketch. We exhibit a natural transformation

$$
\left(\coprod_{\alpha} \circ \bar{T}\right) \circ\left(\xi^{*} \circ \bar{F}\right) \Rightarrow\left(\xi^{*} \circ \bar{F}\right) \circ\left(\coprod_{\alpha} \circ \bar{T}\right) .
$$

This is achieved in Figure 1 by pasting five natural transformations, obtained as follows:

(a) is the counit of the adjunction $\coprod_{\lambda_{X}} \dashv \lambda_{X}^{*}$.

(b) comes from $\bar{\lambda}$ being a lifting of $\lambda$.

(c) comes from the bialgebra condition, the fibration being split, and the units and counits of the adjunctions $\coprod_{\alpha} \dashv \alpha^{*}, \coprod_{F \alpha} \dashv$ $(F \alpha)^{*}$, and $\coprod_{\lambda_{X}} \dashv \lambda_{X}^{*}$

(d) arises since $\bar{T}$ is a lifting of $T$, using the universal property of the Cartesian lifting $(T \xi)^{*}$.

(e) comes from $\bar{F}$ being a lifting of $F$, combined with the unit and counit of the adjunction $\coprod_{\alpha} \dashv \alpha^{*}$.

(Note that like for Theorem 1, this proof actually decomposes into a proof that $\bar{T}$ is $\left(\bar{F}_{\xi},(T \xi)^{*} \circ \lambda_{X}^{*}\right)$-compatible, and a proof that $\coprod_{\alpha}$ is $\left((T \xi)^{*} \circ \lambda_{X}^{*}, \bar{F}_{\xi}\right)$-compatible. $)$

When the fibration at issue is Rel $\rightarrow$ Set and $\bar{T}$ is the canonical lifting $\operatorname{Rel}(T)$, one can easily check that $\coprod_{\alpha} \circ \operatorname{Rel}(T)$ applied to a relation $R$ gives exactly its contextual closure as described in [26]. For this reason, we abbreviate $\coprod_{\alpha} \circ \operatorname{Rel}(T)$ to $C t x$. When moreover $\bar{F}$ is the canonical lifting $\operatorname{Rel}(F)$, we get:

Corollary 3 ([26, Theorem 4]). If F,T are Set-functors and $(X, \alpha, \xi)$ is a bialgebra for $\lambda: T F \Rightarrow F T$. The contextual closure functor $C t x$ is $\operatorname{Rel}(F)_{\xi}$-compatible.

Proof. The canonical lifting $\operatorname{Rel}(-)$ is a 2 -functor [17, Exercise 4.4.6]. Therefore $\bar{\lambda}=\operatorname{Rel}(\lambda)$ fulfils the assumption of Theorem 2 .

Our interest in Theorem 2 is not restricted to prove compatibility of up to $C t x$. By taking non canonical liftings of $T$, one derives novel and effective up-to techniques, such as the monotone contextual closure and the left-contextual closure defined in Sections 5.1 and 5.2. In order to apply Theorem 2 for situations when either $\bar{T}$ or $\bar{F}$ is not the canonical relation lifting, one has to exhibit a $\bar{\lambda}$ sitting above $\lambda$. In Rel, such a $\bar{\lambda}$ exists if and only if for all relations $R \subseteq X^{2}$, the restriction of $\lambda_{X} \times \lambda_{X}$ to $\bar{T} \bar{F} R$ corestricts to $\bar{F} \bar{T} R$. A similar condition has to be checked for Pred $\rightarrow$ Set.

\subsection{Abstract GSOS}

For several applications, it is convenient to consider natural transformations of a slightly different type $\lambda: T(F \times \mathrm{Id}) \Rightarrow F \mathrm{~T}$, where $\mathrm{T}$ is the free monad over $T$. These are called abstract GSOS specifications since, as shown in [31], they generalise GSOS rules to any behaviour endofunctor $F$. Each such $\lambda$ induces a distributive law $\lambda^{\dagger}: \mathrm{T}(F \times \mathrm{Id}) \Rightarrow(F \times \mathrm{Id}) \mathrm{T}$ of the monad $\mathrm{T}$ over the copointed functor $F \times \mathrm{Id}$, whose bialgebras are the objects of our interest (see Appendix B.3). In order to prove compatibility via Theorem 2, one should exhibit a $\overline{\lambda^{\dagger}}$ sitting above $\lambda^{\dagger}$. The following lemma simplifies such a task.

Lemma 1. Let $\bar{\lambda}: \bar{T}(\bar{F} \times \overline{\mathrm{Id}}) \Rightarrow \bar{F} \overline{\mathrm{T}}$ be a natural transformation sitting above $\lambda: T(F \times \mathrm{Id}) \Rightarrow F \mathrm{~T}$. Then there exists a $\overline{\lambda^{\dagger}}: \overline{\mathrm{T}}(\bar{F} \times$ $\overline{\mathrm{Id}}) \Rightarrow(\bar{F} \times \overline{\mathrm{Id}}) \overline{\mathrm{T}}$ sitting above $\lambda^{\dagger}: \mathrm{T}(F \times \mathrm{Id}) \Rightarrow(F \times \mathrm{Id}) \mathrm{T}$.

For a bialgebra $(X, \alpha,\langle\xi, i d\rangle)$, the existence of $\overline{\lambda^{\dagger}}$ ensures, via Theorem 2, compatibility w.r.t. $(\bar{F} \times \mathrm{Id})_{\langle\xi, i d\rangle}$, which is not exactly $\bar{F}_{\xi}$. However, this difference is harmless in poset fibrations: coalgebras for the two functors coincide, and for any pointed functor $A$ compatible with $(\bar{F} \times \mathrm{Id})_{\langle\xi, i d\rangle}$, every $\bar{F}_{\xi}$-invariant up to $A$ is also an $(\bar{F} \times \mathrm{Id})_{\langle\xi, i d\rangle}$-invariant up to $A$. 


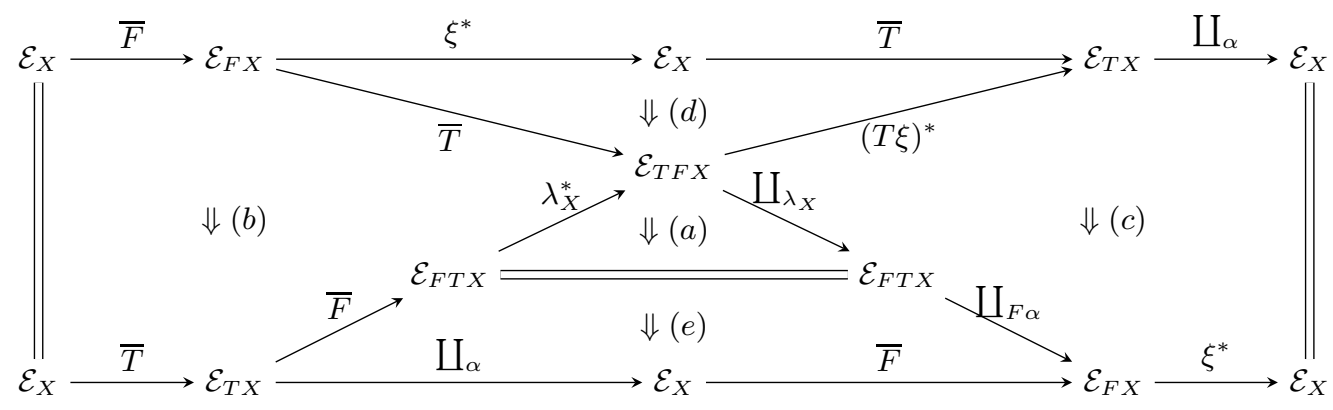

Figure 1. Compatibility of contextual closure in a fibration

\section{Examples}

\subsection{Inclusion of weighted automata}

To illustrate how to instantiate the above framework, we consider weighted automata. We first give a short description of their coalgebraic treatment [6]. For a semiring $\mathrm{S}$ and a set $X$, we denote by $\mathrm{S}_{\omega}^{X}$ the set of functions $f: X \rightarrow \mathrm{S}$ with finite support. These functions can be thought of as linear combinations $\sum_{x \in X} f(x) \cdot x$, and in fact $\mathrm{S}_{\omega}^{-}$: Set $\rightarrow$ Set is the monad sending each set $X$ to the free semi-module generated by $X$.

A weighted automaton over a semiring $\mathrm{S}$ with alphabet $A$ is a pair $(X,\langle o, t\rangle)$, where $X$ is a set of states, $o: X \rightarrow \mathrm{S}$ is an output function associating to each state its output weight and $t: X \rightarrow\left(\mathrm{S}_{\omega}^{X}\right)^{A}$ is a weighted transition relation. Denoting by $F$ the functor $\mathrm{S} \times(-)^{A}$, weighted automata are thus coalgebras for the composite functor $F \mathrm{~S}_{\omega}^{-}$. By the generalised powerset construction [28], they induce bialgebras for the functor $F$, the monad $\mathrm{S}_{\omega}^{-}$, and the distributive law $\lambda: \mathrm{S}_{\omega}^{-} F \Rightarrow F \mathrm{~S}_{\omega}^{-}$given for all sets $X$ by $\lambda_{X}\left(\sum r_{i}\left(s_{i}, \varphi_{i}\right)\right)=\left\langle\sum r_{i} s_{i}, \lambda a . \sum r_{i} \varphi_{i}(a)\right\rangle$. Indeed every $(X,\langle o, t\rangle)$ induces a bialgebra $\left(\mathrm{S}_{\omega}^{X}, \mu,\left\langle o^{\sharp}, t^{\sharp}\right\rangle\right)$ where $\mu$ is the multiplication of $\mathrm{S}_{\omega}^{-}$and $\left\langle o^{\sharp}, t^{\sharp}\right\rangle: \mathrm{S}_{\omega}^{X} \rightarrow \mathrm{S} \times\left(\mathrm{S}_{\omega}^{X}\right)^{A}$ is the linear extension of $\langle o, t\rangle$, defined as $(F \mu) \circ \lambda \circ\left(\mathrm{S}_{\omega}^{\langle o, t\rangle}\right)$.

For a concrete example we take the semiring $\mathrm{R}^{+}$of positive real numbers. A weighted automaton is depicted on the left below: arrows $x \stackrel{a, r}{\rightarrow} y$ mean that $t(x)(a)(y)=r$ and arrows $x \stackrel{r}{\Rightarrow}$ mean that $o(x)=r$.

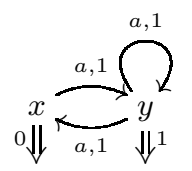

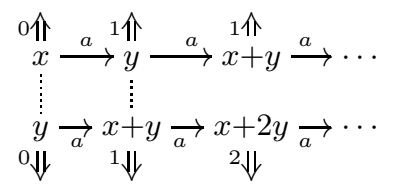

On the right is depicted (part of) the corresponding bialgebra: states are elements of $\left(\mathrm{R}^{+}\right)_{\omega}^{X}$ (hereafter denoted by $v, w$ ), arrows $v \stackrel{a}{\rightarrow} w$ mean that $t^{\sharp}(v)(a)=w$ and arrows $v \stackrel{r}{\Rightarrow}$ mean that $o^{\sharp}(v)=r$.

Whenever $\mathrm{S}$ carries a partial order $\leq$, one can take the following lifting $\bar{F}:$ Rel $\rightarrow$ Rel of $F$ defined for $R \subseteq X^{2}$ by:

$$
\{((r, \varphi),(s, \psi)) \mid r \leq s \wedge \forall a \cdot \varphi(a) R \psi(a)\} \subseteq(F X)^{2} .
$$

Then the functor $\bar{F}_{\left\langle o^{\sharp}, t^{\sharp}\right\rangle}=\left\langle o^{\sharp}, t^{\sharp}\right\rangle^{*} \circ \bar{F}: \operatorname{Rel}_{X} \rightarrow \operatorname{Rel}_{X}$ maps a relation $R \subseteq X^{2}$ into

$$
\left\{(x, y) \mid o^{\sharp}(x) \leq o^{\sharp}(y) \wedge \forall a \cdot t^{\sharp}(x)(a) R t^{\sharp}(y)(a)\right\} .
$$

The carrier of a final $\bar{F}_{\left\langle o^{\sharp}, t^{\sharp}\right\rangle}$-coalgebra is a relation, denoted by $\precsim$, which we call inclusion: when $\mathrm{S}$ is the Boolean semiring, it coincides with language inclusion of non-deterministic automata.

For any two $v, w \in \mathrm{S}_{\omega}^{X}$, one can prove that $v \precsim w$ by exhibiting a $\bar{F}_{\left\langle o^{\sharp}, t \sharp\right\rangle}$-invariant relating them. These invariants are usually infinite, since there are infinitely many reachable states in a bialgebra $\mathrm{S}_{\omega}^{X}$, even for finite $X$. This is the case when trying to check $x \precsim y$ in (1): we should relate infinitely many reachable states.

In order to obtain finite proofs, we exploit the algebraic structure of bialgebras and employ an up to context technique. To this end, we use the canonical lifting of the monad $S_{\omega}^{-}$, defined for all $R \subseteq X^{2}$ as

$$
\operatorname{Rel}\left(\mathrm{S}_{\omega}^{-}\right)(R)=\left\{\left(\sum r_{i} x_{i}, \sum r_{i} y_{i}\right) \mid x_{i} R y_{i}\right\}
$$

We prove that the endofunctor $C t x=\coprod_{\mu} \circ \operatorname{Rel}\left(S_{\omega}^{-}\right)$is $\bar{F}_{\left\langle o^{\sharp}, t^{\sharp}\right\rangle}$ compatible by Theorem 2: it suffices to check that for any relation $R$ on $X$, the restriction of $\lambda_{X} \times \lambda_{X}$ to $\operatorname{Rel}\left(\mathrm{S}_{\omega}^{-}\right) \bar{F}(R)$ corestricts to $\bar{F} \operatorname{Rel}\left(\mathrm{S}_{\omega}^{-}\right)(R)$. This is the case when for all $n_{1}, m_{1}, n_{2}, m_{2} \in \mathrm{S}$ such that $n_{1} \leq m_{1}$ and $n_{2} \leq m_{2}$, we have (a) $n_{1}+n_{2} \leq m_{1}+m_{2}$ and (b) $n_{1} \cdot n_{2} \leq m_{1} \cdot m_{2}$. These two conditions are satisfied, e.g., in the Boolean semiring or in $\mathrm{R}^{+}$and thus, in these cases, we can prove inclusion of automata using $\bar{F}_{\left\langle o^{\sharp}, t \sharp\right\rangle}$-invariants up to $C t x$. For example, in (1), the relation $R=\{(x, y),(y, x+y)\}$ is a $\bar{F}_{\left\langle o^{\sharp}, t \sharp\right.}$-invariant up to $C t x$ (to check this, just observe that $(x+y, x+2 y) \in C t x(R))$. This finite relation thus proves $x \precsim y$.

Unfortunately, condition (b) fails for the semiring $\mathrm{R}$ of (all) real numbers. Nevertheless, our framework allows us to define another up-to technique, which we call "up to monotone contextual closure". It is obtained by composing $\coprod_{\mu}$ and a non-canonical lifting of $\mathrm{R}_{\omega}^{-}$:

$$
\overline{\mathrm{R}_{\bar{\omega}}}(R)=\left\{\left(\sum r_{i} x_{i}, \sum r_{i} y_{i}\right) \mid \begin{array}{l}
r_{i} \geq 0 \Rightarrow x_{i} R y_{i} \\
r_{i}<0 \Rightarrow y_{i} R x_{i}
\end{array}\right\}
$$

The restriction of $\lambda_{X} \times \lambda_{X}$ to $\overline{\mathrm{R}_{\omega}^{-}} \bar{F}(R)$ corestricts to $\overline{\bar{F} \overline{\mathrm{R}_{\omega}}}(R)$. Therefore, by Theorem 2 , the monotone contextual closure is $\bar{F}_{\left\langle o^{\sharp}, t^{\sharp}\right\rangle}$-compatible.

\subsection{Divergence of processes}

Up-to techniques can be instrumental in proving unary predicates. We take the fibration Pred $\rightarrow$ Set and we focus on the divergence predicate $\nu u .\langle\tau\rangle u$ defined on LTSs. The latter are coalgebras $\xi: X \rightarrow F(X)$ for the Set-functor $F X=\mathcal{P}_{\omega}(L \times X)$, where $L=\{a, \bar{a}, b, \bar{b} \ldots, \tau\}$ is a set of labels containing a special symbol $\tau$ and $\mathcal{P}_{\omega}$ is the finite powerset functor. We lift $F$ to $\bar{F}^{\langle\tau\rangle}:$ Pred $\rightarrow$ Pred, defined for all sets $X$ as

$$
\bar{F}_{X}^{\langle\tau\rangle}(P \subseteq X)=\{S \in F X \mid \exists(\tau, x) \in S, x \in P\} .
$$

The final $\bar{F}_{\xi}^{\langle\tau\rangle}$-coalgebra consists precisely of all the states in $X$ satisfying $\nu u .\langle\tau\rangle u$. Hence, to prove that a state $p$ diverges, it suffices to exhibit an $\bar{F}_{\xi}^{\langle\tau\rangle}$-invariant containing $p$. 
When the LTS is specified by some process algebra, such invariants might be infinite. Suppose for instance that we have a parallel operator defined by the following GSOS rules and their symmetric counterparts:

$$
\frac{x \stackrel{l}{\rightarrow} x^{\prime}}{x\left|y \stackrel{l}{\rightarrow} x^{\prime}\right| y} \quad \frac{x \stackrel{a}{\rightarrow} x^{\prime} \quad y \stackrel{\bar{a}}{\rightarrow} y^{\prime}}{x\left|y \stackrel{\tau}{\rightarrow} x^{\prime}\right| y^{\prime}} .
$$

Consider the processes $p \stackrel{a}{\rightarrow} p \mid p$ and $q \stackrel{\bar{a}}{\rightarrow} q$. To prove that $p \mid q$ diverges, any invariant should include all the states that are on the infinite path $p|q \stackrel{\tau}{\rightarrow}(p \mid p)| q \stackrel{\tau}{\rightarrow} \ldots$. .

Instead, an intuitive proof would go as follows: assuming that $p \mid q$ diverges one has to prove that the $\tau$ successor $(p \mid p) \mid q$ also diverges. Rather than looking further for the $\tau$-successors of $(p \mid p) \mid q$, observe that

(a) since $p \mid q$ diverges by hypothesis, then also $(p \mid q) \mid p$ diverges, and

(b) since $(p \mid q) \mid p$ is bisimilar (i.e., behavioural equivalent) to $(p \mid p) \mid q$, then also $(p \mid p) \mid q$ diverges.

Formally, (b) corresponds to using the functor Bhv from Section 4.1. For (a) we define the left contextual closure functor as $C t x^{\ell}(P \subseteq X)=\left\{\left(\ldots\left(x \mid y_{1}\right) \mid \ldots\right)\left|y_{n}\right| x \in P, y_{i} \in X\right\}$. Indeed, it is easy to see that $P=\{p \mid q\}$ is an $\bar{F}_{\xi}^{\langle\tau\rangle}$-invariant up to $B h v \circ C t x^{\ell}$, i.e, $P \subseteq \bar{F}_{\xi}^{\langle\tau\rangle} \circ B h v \circ \operatorname{Ctx}^{\ell}(P)$.

In order to prove soundness of this "up to behavioural equivalence and left contextual closure", we show compatibility of Bhv and $C t x^{\ell}$ separately. For the former, we note that $\bar{F}^{\langle\tau\rangle}$ is defined exactly as in coalgebraic modal logic $[9,13]$ and thus $\left(\bar{F}^{\langle\tau\rangle}, F\right)$ is a fibration map: Theorem 1 applies. The functor $C t x^{\ell}$ is defined just as $C t x$, but instead of the canonical lifting of the endofunctor for binary operations $T(X)=X \times X$ we use the predicate lifting $\bar{T}(P \subseteq X)=P \times X \subseteq T X$. The conditions of Lemma 1 are met for the distributive law given by the above GSOS rules (see Appendix C). The functor $C t x^{\ell}$ can be seen to be the composition $\coprod_{\mu} \circ \overline{\mathrm{T}}$ where $\overline{\mathrm{T}}$ is the free monad on $\bar{T}$ and $\mu$ is the multiplication of $\mathrm{T}$. We can thus apply Theorem 2 and obtain its compatibility.

\subsection{Equivalence of nominal automata}

Nominal automata and variants [4] have been considered as a means of studying languages over infinite alphabets, but also for the operational semantics of process calculi [22]. We refer the reader to [23] for background on the category Nom of nominal sets. These are sets equipped with actions of the group of permutations on a countable set $\mathrm{A}$ of names, satisfying an additional finite support condition.

Consider the nominal automaton below. The part reachable from state $*$ corresponds to [5, Example I.1].

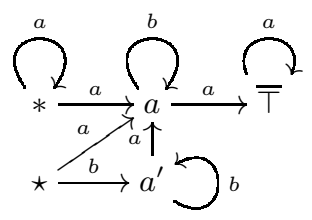

It is important to specify how to read this drawing: the represented nominal automaton has as state space the orbit-finite nominal set $\{*\}+\{\star\}+A+A^{\prime}+\{T\}$, where $A^{\prime}$ is a copy of $A$. It suffices in this case to give only one representative of each of the five orbits: we span all the transitions and states of the automaton by applying all possible finite permutations to those explicitly written. For example, the transition $a \stackrel{c}{\rightarrow} a$ is obtained from $a \stackrel{b}{\rightarrow} a$ by applying the transposition $(b c)$ to the latter.
With this semantics in mind, one can see that the state $*$ accepts the language of words in the alphabet $A$ where some letter appears twice: it reads a word in $A$, then it nondeterministically guesses that the next letter will appear a second time and verifies that this is indeed the case. The state $\star$ accepts the same language, in a different way: it reads a first letter, then guesses if this letter will be read again, or, if a distinct letter-nondeterministically chosenwill appear twice.

Formally, nominal automata are $F \mathcal{P}_{\omega}$-coalgebras $\langle o, t\rangle$ where $F:$ Nom $\rightarrow$ Nom is given by $F X=2 \times X^{\mathbb{A}}$ and the monad $\mathcal{P}_{\omega}$ is the finitary version of the power object functor in the category of nominal sets (mapping a nominal set to its finitely-supported orbitfinite subsets). In our example, $o(a)=0$ and $t(a)$ is the following map:

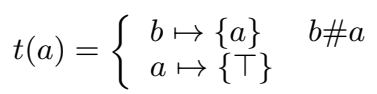

By the generalised powerset construction [28], $\langle o, t\rangle$ induces a deterministic nominal automata, which is a bialgebra on $\mathcal{P}_{\omega}(X)$ with the algebraic structure given by union. To prove that $*$ and $\star$ accept the same language, we should play the bisimulation game in the determinisation of the automaton. However, the latter has infinitely many orbits and a rather complicated structure. A bisimulation constructed like this will thus have infinitely many orbits. Instead, we can show that the orbit-finite relation spanned by the four pairs

$$
(\{*\},\{\star\}),\left(\{a\},\left\{a, a^{\prime}\right\}\right),(\{\top\},\{a, \top\}),\left(\{*\}, \mathrm{A}^{\prime}\right)
$$

is a bisimulation up to congruence (w.r.t. union).

The soundness of this technique is established in Appendix D using the fibration $\operatorname{Rel}(\mathrm{Nom}) \rightarrow$ Nom of equivariant relations. We derive the compatibility of contextual closure using Theorem 2 , and compatibility of the transitive, symmetric, and reflexive closures using Proposition 2. Compatibility of congruence closure follows from Proposition 1(i).

\section{Compositional Predicates}

In this section we consider a structured way of defining coinductive predicates, by composing lifted functors. Assume a fibration $p: \mathcal{E} \rightarrow \mathcal{B}$ and a functor $\otimes: \mathcal{E} \times_{\mathcal{B}} \mathcal{E} \rightarrow \mathcal{E}$. Given two liftings $\overline{F_{1}}, \overline{F_{2}}: \mathcal{E} \rightarrow \mathcal{E}$ of the same endofunctor $F$ on $\mathcal{B}$, one can then define a composite lifting $\otimes \circ\left\langle\overline{F_{1}}, \overline{F_{2}}\right\rangle$, which we denote by $\overline{F_{1}} \otimes \overline{F_{2}}$. We will instantiate this to the fibration Rel $\rightarrow$ Set with relational composition for $\otimes$, to define simulation and weak bisimulation as coinductive predicates.

One advantage of this approach is that the compatibility of upto-context can be proved in a modular way.

Theorem 3. Let $\bar{T}$ be a lifting of T having a $\gamma: \bar{T} \otimes \Rightarrow \otimes \bar{T}^{2}$ above Id: $T \Rightarrow T$. Let both $\overline{F_{1}}$ and $\overline{F_{2}}$ be liftings of $F$. If $\lambda_{1}: \bar{T} \overline{F_{1}} \Rightarrow \overline{F_{1}} \bar{T}$ and $\lambda_{2}: \bar{T} \overline{F_{2}} \Rightarrow \overline{F_{2}} \bar{T}$ sit above the same $\lambda: T F \Rightarrow F T$, then there exists $\bar{\lambda}: \bar{T}\left(\overline{F_{1}} \otimes \overline{F_{2}}\right) \Rightarrow\left(\overline{F_{1}} \otimes \overline{F_{2}}\right) \bar{T}$ above $\lambda$.

Notice that the canonical lifting $\operatorname{Rel}(T)$ always satisfies the first hypothesis of the theorem when $\otimes$ is relational composition.

\subsection{Simulation up-to}

We recall simulations for coalgebras as introduced in [15]. An endofunctor $F$ on Set is said to be ordered if it factors through the forgetful functor from Pre (the category of preorders) to Set: this means that for every $X, F X$ is equipped with a preorder $\sqsubseteq_{F X}$. An ordered functor gives rise to a constant relation lifting $\bar{\Xi}$ of $F$ defined as $\sqsubseteq\left(R \subseteq X^{2}\right)=\sqsubseteq_{F X}$. Then the lax relation lifting 
$\operatorname{Rel}(F) \sqsubseteq$ is defined as

$$
\operatorname{Rel}(F)^{\sqsubseteq}=\sqsubseteq \otimes \operatorname{Rel}(F) \otimes \sqsubseteq
$$

where $\otimes$ is relational composition. For a coalgebra $\xi: X \rightarrow F X$, the coalgebras for the endofunctor $\xi^{*} \circ \operatorname{Rel}(F) \stackrel{ }{\bar{X}}$-which we denote as $\operatorname{Rel}(F) \frac{\sqsubseteq}{\xi}$-are called simulations; the final one is called similarity. We list two examples of ordered functors and their associated notion of simulations, and refer to [15] for many more.

Example 3. For weighted automata on a semiring S equipped with a partial order $\leq$, the functor $F X=\mathrm{S} \times X^{A}$ is ordered with $\sqsubseteq_{F X}$ defined as $(s, \phi) \sqsubseteq_{F X}(r, \psi)$ iff $s \leq r$ and $\phi=\psi$. It is immediate to see that $\operatorname{Rel}(F) \sqsubseteq$ coincides with the lifting $\bar{F}$ defined in Section 5.1.

For LTSs, the functor $F X=\mathcal{P}_{\omega}(A \times X)$ is ordered with subset inclusion $\subseteq$. In this case a simulation is a relation $R \subseteq X^{2}$ such that for all $(x, y) \in R$ : if $x \stackrel{a}{\rightarrow} x^{\prime}$ then there exists $y^{\prime}$ such that $x^{\prime} \stackrel{a}{\rightarrow} y^{\prime}$ and $x^{\prime} R y^{\prime}$.

An ordered functor $F$ is called stable if $\left(\operatorname{Rel}(F)^{\sqsubseteq}, F\right)$ is a fibration map [15]. Since polynomial functors are stable, as well as the one for LTSs [15], the following results hold for the coalgebras in Example 3.

Proposition 3. If $F$ is a stable ordered functor, then Bhv, Slf, and

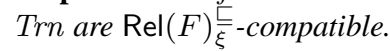

Proof. Compatibility of Bhv comes from Theorem 1. Compatibility of Slf and Trn comes from Proposition 2: stable functors satisfy $(* * *)[15$, Lemma 5.3].

We proceed to consider the compatibility of up to context, for which we assume an abstract GSOS specification $\lambda: T(F \times I d) \Rightarrow$ $F$ T. By Theorem 3, proving compatibility w.r.t. $\operatorname{Rel}(F) \frac{\complement}{\xi}$ is reduced to proving compatibility w.r.t. its components $\operatorname{Rel}(F)$ and 巨. For the former, compatibility comes immediately from the proof of Corollary 3. For the latter, we need to assume that the abstract GSOS specification is monotone, i.e, such that for any set $X$, the restriction of $\lambda_{X} \times \lambda_{X}$ to $\operatorname{Rel}(T)\left(\sqsubseteq_{F X} \times \Delta_{X}\right)$ corestricts to $\Xi_{F \pi X}$. If $T$ is a polynomial functor representing a signature, then this means that for any operator $\sigma$ (of arity $n$ ) we have

$$
\frac{b_{1} \sqsubseteq_{F X} c_{1} \quad \ldots \quad b_{n} \sqsubseteq_{F X} c_{n}}{\lambda_{X}(\sigma(\mathbf{b}, \mathbf{x})) \sqsubseteq_{F \mathbb{} X} \lambda_{X}(\sigma(\mathbf{c}, \mathbf{x}))}
$$

where $\mathbf{b}, \mathbf{x}=\left(b_{1}, x_{1}\right), \ldots,\left(b_{n}, x_{n}\right)$ with $x_{i} \in X$ and similarly for $\mathbf{c}, \mathbf{x}$. If $\sqsubseteq$ is the order on the functor for LTSs, monotonicity corresponds to the positive GSOS format [12] which, as expected, is GSOS [3] without negative premises. Monotonicity turns out to be precisely the condition needed to apply Lemma 1, yielding

Proposition 4. Let $\lambda$ be a monotone abstract GSOS specification and $(X, \alpha,\langle\xi, i d\rangle)$ be a $\lambda^{\dagger}$-bialgebra. Then Ctx is $\left(\operatorname{Rel}(F)^{\sqsubseteq} \times\right.$ Id $)_{\langle\xi, i d\rangle}$-compatible.

\subsection{Weak bisimulation-up-to}

A weak bisimulation is a relation $R \subseteq X^{2}$ on the states of an LTS such that for every pair $(x, y) \in R$ : (1) if $x \stackrel{l}{\rightarrow} x^{\prime}$ then $y \stackrel{l}{\Rightarrow} y^{\prime}$ with $\left(x^{\prime}, y^{\prime}\right) \in R$ and (2) if $y \stackrel{l}{\rightarrow} y^{\prime}$ then $x \stackrel{l}{\Rightarrow} x^{\prime}$ with $\left(x^{\prime}, y^{\prime}\right) \in R$. Here $\rightarrow$ and $\Rightarrow$ are two LTSs, i.e., coalgebras for the functor $F X=\mathcal{P}_{\omega}(L \times X)$, and $\Rightarrow$ is the saturation [21] of $\rightarrow$. Weak bisimilarity can alternatively be reduced to strong bisimilarity on $\Rightarrow$, but the associated proof method is rather tedious. To remain faithful to the above definition, we define weak bisimulations via the following lifting of $F \times F$ :

$$
\overline{F \times F}=\rho \otimes \operatorname{Rel}(F \times F)^{[\supseteq \subseteq]},
$$

where $\rho$ is the constant functor defined as $\rho\left(R \subseteq X^{2}\right)=$ $\{((U, V),(V, U)) \mid U, V \in F X\}$ and $\operatorname{Rel}(F \times F)^{\left[\supseteq{ }^{[\subseteq}\right]}$ is the lax relation lifting of $F \times F$ for the ordering $\left(U_{1}, V_{1}\right)[\supseteq \subseteq]\left(U_{2}, V_{2}\right)$ iff $U_{2} \subseteq U_{1}$ and $V_{1} \subseteq V_{2}$.

For an intuition, observe that an $F \times F$-coalgebra is a pair $\left\langle\xi_{1}, \xi_{2}\right\rangle: X \rightarrow F X \times F X$ of LTSs that we denote with $\rightarrow_{1}$ and $\rightarrow_{2}$. An invariant for $\operatorname{Rel}(F \times F)_{\left\langle\xi_{1}, \xi_{2}\right\rangle}^{[\supseteq \subseteq]}$ is a relation $R \subseteq X^{2}$ such that for each $(x, y) \in R$ : (1) if $y \stackrel{l}{\rightarrow}_{1} y^{\prime}$ then $x \stackrel{l}{\rightarrow}_{1} x^{\prime}$ with $x^{\prime} R y^{\prime}$, and (2) if $x \stackrel{l}{\rightarrow}_{2} x^{\prime}$ then $y \stackrel{l}{\rightarrow}_{2} y^{\prime}$ with $x^{\prime} R y^{\prime}$. Composing with $\rho$ "flips" the LTSs $\rightarrow_{1}$ and $\rightarrow_{2}$ : an invariant for $\overline{F \times F}\left\langle\xi_{1}, \xi_{2}\right\rangle$ is now an $R \subseteq X^{2}$ such that: (1) if $y \stackrel{l}{\rightarrow}_{1} y^{\prime}$ then $x \stackrel{l}{\rightarrow}_{2} x^{\prime}$ with $x^{\prime} R y^{\prime}$, and (2) if $x \stackrel{l}{\rightarrow}_{1} x^{\prime}$ then $y \stackrel{l}{\rightarrow}_{2} y^{\prime}$ with $x^{\prime} R y^{\prime}$. It is easy to see that for $\left\langle\xi_{1}, \xi_{2}\right\rangle=\langle\rightarrow, \Rightarrow\rangle$, coalgebras for $\overline{F \times F}\left\langle\xi_{1}, \xi_{2}\right\rangle$ are weak bisimulations and the final coalgebra is weak bisimilarity.

In Appendix E, we show that $(\overline{F \times F}, F)$ is a fibration map and by Theorem 1 we now obtain the following.

Corollary 4. Bhv is $\overline{F \times F}_{\left\langle\xi_{1}, \xi_{2}\right\rangle}$-compatible.

For $\left\langle\xi_{1}, \xi_{2}\right\rangle=\langle\rightarrow, \Rightarrow\rangle$, behavioural equivalence is simply strong bisimilarity. Consequently, Corollary 4 actually gives the compatibility of weak bisimulation up to strong bisimilarity [25]. One could wish to use up to Slf or up to Trn for weak bisimulations. However, the condition (***) from Section 4.2 fails, and indeed, weak bisimulations up to weak bisimilarity or up to transitivity are not sound [25].

For up to context, we use Theorem 3 to reduce compatibility w.r.t. $\overline{F \times F}$ to compatibility w.r.t. $\rho$ and $\operatorname{Rel}(F \times F)^{[\supseteq \subseteq]}$ (for which we can reuse the result of the previous section).

Proposition 5. Let $\lambda: T(F \times \mathrm{Id}) \Rightarrow F \mathrm{~T}$ be a positive GSOS specification and $\left(X, \alpha,\left\langle\xi_{1}, i d\right\rangle\right)$ and $\left(X, \alpha,\left\langle\xi_{2}, i d\right\rangle\right)$ be two $\lambda^{\dagger}$ bialgebras then Ctx is $(\overline{F \times F} \times \mathrm{Id})_{\left\langle\xi_{1}, \xi_{2}, i d\right\rangle}$-compatible.

The above proposition requires both $\rightarrow$ and $\Rightarrow$ to be models [1] of the same positive GSOS specification $\lambda$. This means that the rules of $\lambda$ should be sound for both $\rightarrow$ and $\Rightarrow$. For instance, in the case of $\mathrm{CCS}, \Rightarrow$ is not a model of $\lambda$ because the rule for nondeterministic choice is not sound for $\Rightarrow$. Nevertheless, we can use our framework to prove the compatibility of weak bisimulation up to contextual closure w.r.t. the remaining operators.

\section{Directions for future work}

Our nominal automata example leads us to expect that the framework introduced in this paper will lend itself to obtaining a clean theory of up-to techniques for name-passing process calculi. For instance, we would like to understand whether the congruence rule format proposed by Fiore and Staton [11] can fit in our setting: this would provide general conditions under which up-to techniques related to name substitution are sound in such calculi.

Another interesting research direction is suggested by the divergence predicate we studied in Section 5.2. Other formulas of (coalgebraic) modal logic [9] can be expressed by taking different predicate liftings, and yield different families of compatible functors. This suggests a connection with the proof systems in [10, 29]: we can regard proofs in those systems as invariants up to some compatible functors. By using our framework and the logical distributive laws of [18], we hope to obtain a systematic way to derive or enhance such proof systems, starting from a given abstract GSOS specification.

\section{Acknowledgments}

We are grateful to the anonymous reviewers for their constructive comments, and in particular to the one who noticed that our no- 
tion of compatible functor was just an instance of morphisms of endofunctors. We would also like to thank Alexander Kurz and Alexandra Silva for the stimulating discussions that eventually led to the example on nominal automata; Marcello Bonsangue, Tom Hirschowitz and Henning Kerstan for comments on preliminary versions of the paper; Ichiro Hasuo for the inspiring talk at Bellairs Workshop on Coalgebras.

\section{References}

[1] L. Aceto, W. Fokkink, and C. Verhoef. Structural operational semantics. In Handbook of Process Algebra, pages 197-292. Elsevier, 2001.

[2] F. Bartels. Generalised coinduction. MSCS, 13(2):321-348, 2003.

[3] B. Bloom, S. Istrail, and A. R. Meyer. Bisimulation can't be traced. In POPL, pages 229-239. ACM, 1988.

[4] M. Bojanczyk, B. Klin, and S. Lasota. Automata with group actions. In LICS, pages 355-364, 2011.

[5] M. Bojanczyk, B. Klin, S. Lasota, and S. Torunczyk. Turing machines with atoms. In LICS, pages 183-192, 2013.

[6] F. Bonchi, M. Bonsangue, M. Boreale, J. Rutten, and A. Silva. A coalgebraic perspective on linear weighted automata. Inf. and Comp., 211:77-105, 2012.

[7] F. Bonchi and D. Pous. Checking NFA equivalence with bisimulations up to congruence. In POPL, pages 457-468. ACM, 2013.

[8] D. Caucal. Graphes canoniques de graphes algébriques. ITA, 24:339$352,1990$.

[9] C. Cîrstea, A. Kurz, D. Pattinson, L. Schröder, and Y. Venema. Modal logics are coalgebraic. Comput. J., 54(1):31-41, 2011.

[10] M. Dam. Compositional proof systems for model checking infinite state processes. In CONCUR, volume 962 of LNCS, pages 12-26. Springer, 1995

[11] M. Fiore and S. Staton. A congruence rule format for name-passing process calculi. Inf. and Comp., 207(2):209-236, 2009.

[12] M. Fiore and S. Staton. Positive structural operational semantics and monotone distributive laws. In CMCS, page 8, 2010.

[13] I. Hasuo, K. Cho, T. Kataoka, and B. Jacobs. Coinductive predicates and final sequences in a fibration. In MFPS, 2013.

[14] C. Hermida and B. Jacobs. Structural induction and coinduction in a fibrational setting. Inf. and Comp., 145:107-152, 1997.

[15] J. Hughes and B. Jacobs. Simulations in coalgebra. TCS, 327(1-2):71108, 2004.

[16] B. Jacobs. Categorical Logic and Type Theory. Elsevier, 1999.

[17] B. Jacobs. Introduction to coalgebra. Towards mathematics of states and observations, 2014. Draft.

[18] B. Klin. Bialgebraic operational semantics and modal logic. In LICS, pages 336-345. IEEE, 2007.

[19] M. Lenisa. From set-theoretic coinduction to coalgebraic coinduction: some results, some problems. ENTCS, 19:2-22, 1999.

[20] M. Lenisa, J. Power, and H. Watanabe. Distributivity for endofunctors, pointed and co-pointed endofunctors, monads and comonads. ENTCS, 33:230-260, 2000.

[21] R. Milner. Communication and Concurrency. Prentice Hall, 1989.

[22] U. Montanari and M. Pistore. History-dependent automata: An introduction. In SFM, LNCS, pages 1-28. Springer, 2005.

[23] A. M. Pitts. Nominal Sets. Cambridge University Press, 2013.

[24] D. Pous. Complete lattices and up-to techniques. In APLAS, volume 4807 of $L N C S$, pages 351-366. Springer, 2007.

[25] D. Pous and D. Sangiorgi. Enhancements of the bisimulation proof method. In Advanced Topics in Bisimulation and Coinduction, pages 233-289. Cambridge University Press, 2012.

[26] J. Rot, F. Bonchi, M. Bonsangue, D. Pous, J. Rutten, and A. Silva. Enhanced coalgebraic bisimulation. To appear in MSCS, 2014. Available at http://www.liacs.nl/ jrot/up-to.pdf.
[27] D. Sangiorgi. On the bisimulation proof method. MSCS, 8:447-479, 1998.

[28] A. Silva, F. Bonchi, M. Bonsangue, and J. Rutten. Generalizing the powerset construction, coalgebraically. In FSTTCS, pages 272-283, 2010.

[29] A. Simpson. Sequent calculi for process verification: HennessyMilner logic for an arbitrary GSOS. JLAP, 60-61:287-322, 2004.

[30] S. Staton. Relating coalgebraic notions of bisimulation. LMCS, 7(1), 2011.

[31] D. Turi and G. D. Plotkin. Towards a mathematical operational semantics. In LICS, pages 280-291. IEEE, 1997. 


\section{A. Proofs for Section 3}

The following Proposition generalises the compositionality results for compatible functions on lattices, see [24] or [25, Proposition 6.3.11].

Proposition 1. Compatible functors are closed under the following constructions:

(i) composition: if $A$ is $(B, C)$-compatible and $A^{\prime}$ is $(C, D)$ compatible, then $A^{\prime} \circ A$ is $(B, D)$-compatible;

(ii) pairing: if $\left(A_{i}\right)_{i \in \iota}$ are $(B, C)$-compatible, then $\left\langle A_{i}\right\rangle_{i \in \iota}$ is $\left(B, C^{\iota}\right)$-compatible;

(iii) product: if $A$ is $(B, C)$-compatible and $A^{\prime}$ is $\left(B^{\prime}, C^{\prime}\right)$ compatible, then $A \times A^{\prime}$ is $\left(B \times B^{\prime}, C \times C^{\prime}\right)$-compatible;

Moreover, for an endofunctor $B: \mathcal{C} \rightarrow \mathcal{C}$,

(iv) the identity functor $I d: \mathcal{C} \rightarrow \mathcal{C}$ is $B$-compatible;

(v) the constant functor to the carrier of any B-coalgebra is Bcompatible, in particular the final one if it exists;

(vi) the coproduct functor $\amalg: \mathcal{C}^{\iota} \rightarrow \mathcal{C}$ is $\left(B^{\iota}, B\right)$-compatible.

Proof. (i) Given $\gamma: A B \Rightarrow C A$ and $\gamma^{\prime}: A^{\prime} C \Rightarrow D A^{\prime}$ we obtain

$$
A^{\prime} A B \stackrel{A^{\prime} \gamma}{\Longrightarrow} A^{\prime} C A \stackrel{\gamma^{\prime} A}{\Longrightarrow} D A^{\prime} A
$$

(ii) Given natural transformations $\gamma_{i}: A_{i} B \Rightarrow C A_{i}$ for all $i \in \iota$ we obtain a natural transformation

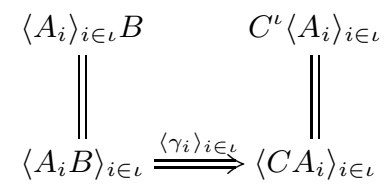

(iii) Given $\gamma: A B \Rightarrow C A$ and $\gamma^{\prime}: A^{\prime} B^{\prime} \Rightarrow C^{\prime} A^{\prime}$ we construct $\gamma \times \gamma^{\prime}:\left(A \times A^{\prime}\right)\left(B \times B^{\prime}\right) \Rightarrow\left(C \times C^{\prime}\right)\left(A \times A^{\prime}\right)$.

Items (iv), (v) and (vi) are trivial. For example, the latter is immediate using the universal property of the coproduct.

\section{B. Proofs for Section 4}

The next simple Lemma about liftings in fibrations will be used throughout this appendix, e.g., to prove Proposition 2, but also Theorem 2.

Lemma 2. Let $p: \mathcal{E} \rightarrow \mathcal{B}$ and $p^{\prime}: \mathcal{E}^{\prime} \rightarrow \mathcal{B}$ be two fibrations and assume $\bar{T}: \mathcal{E} \rightarrow \mathcal{E}^{\prime}$ is the lifting of a functor $T: \mathcal{B} \rightarrow \mathcal{B}$. Consider a $\mathcal{B}$-morphism $f: X \rightarrow Y$. Then there exists a natural transformation:

$$
\theta: \bar{T} \circ f^{*} \Rightarrow(T f)^{*} \circ \bar{T}: \mathcal{E}_{Y} \rightarrow \mathcal{E}_{T X}^{\prime} .
$$

Proof. In order to define $\theta_{R}$ for some $R$ in $\mathcal{E}_{Y}$, we use the universal property of the Cartesian lifting $\widetilde{T f} f_{\bar{T}(R)}$. In a diagram:

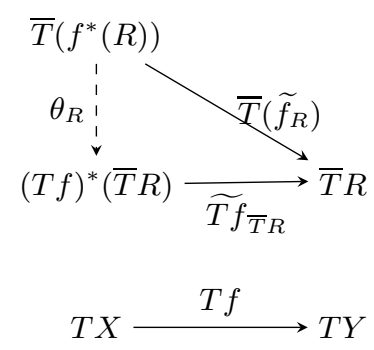

Lemma 3. Let $p: \mathcal{E} \rightarrow \mathcal{B}$ be a bifibration and assume $\bar{F}: \mathcal{E} \rightarrow \mathcal{E}$ is the lifting of a functor $F: \mathcal{B} \rightarrow \mathcal{B}$. Consider a $\mathcal{B}$-morphism $f: X \rightarrow Y$. Then there exists a natural transformation:

$$
\rho: \coprod_{F f} \circ \bar{F} \Rightarrow \bar{F} \circ \coprod_{f}: \mathcal{E}_{X} \rightarrow \mathcal{E}_{F Y} .
$$

Proof. The proof uses the universal property of the opcartesian liftings. Equivalently, from Lemma 2 we have a natural transformation $\bar{F} f^{*} \Rightarrow(F f)^{*} \bar{F}$. Taking the adjoint transpose via $\coprod_{F f} \dashv(F f)^{*}$ we get a natural transformation $\coprod_{F f} \bar{F} f^{*} \Rightarrow \bar{F}$. A further adjoint transpose via the adjunction $\coprod_{f} \dashv f^{*}$ yields the desired $\rho: \coprod_{F f} \bar{F} \Rightarrow \bar{F} \coprod_{f}$.

\section{B.1 Proofs for Section 4.2}

In this section we prove Proposition 2. For the sake of clarity we explain how $\bar{F}^{n}$ is defined for $n=2$. Recall that $\mathcal{E} \times{ }_{\mathcal{B}} \mathcal{E}$ is obtained as a pullback of $p$ along $p$ in Cat.

For a lifting $\bar{F}$ of $F$, the functor $\bar{F}^{2}$ makes the next diagram commute.

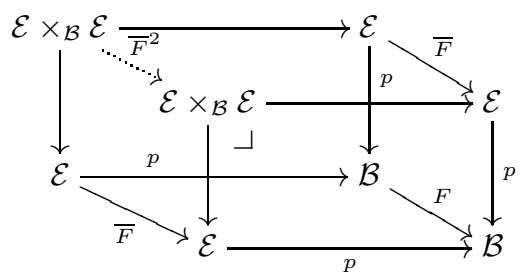

This means that on each fibre we have

$$
\bar{F}_{X}^{n}=\left(\bar{F}_{X}\right)^{n}: \mathcal{E}_{X}^{n} \rightarrow \mathcal{E}_{F X}^{n} .
$$

As a consequence of Lemma 2 we obtain:

Lemma 4. Let $p: \mathcal{E} \rightarrow \mathcal{B}$ and assume $G: \mathcal{E}^{\times{ }_{\mathcal{B}} n} \rightarrow \mathcal{E}$ is a lifting of the identity on $\mathcal{B}$. If $f: X \rightarrow Y$ is a $\mathcal{B}$-morphism, there is a canonical natural transformation

$$
\theta: G\left(f^{*}\right)^{n} \Rightarrow f^{*} G: \mathcal{E}_{Y} \rightarrow \mathcal{E}_{G X}
$$

Proof. This is an instance of Lemma 2 for $T=\operatorname{Id}$ and $\bar{T}=G$. We also use that the Cartesian lifting of a $\mathcal{B}$-morphism $f$ in $\mathcal{E}^{\times \mathcal{B}^{n}}$ is $\left(f^{*}\right)^{n}$, where $f^{*}$ is the Cartesian lifting in $\mathcal{E}$.

Proposition 2. Let $G: \mathcal{E}^{\times \mathcal{B}^{n}} \rightarrow \mathcal{E}$ be a lifting of the identity on $\mathcal{B}$ such that there exists a natural transformation $G \bar{F}^{n} \Rightarrow \bar{F} G$. Then $G_{X}$ is $\bar{F}_{\xi \text {-compatible. }}$

Proof. Consider the natural transformation obtained as the composition

$$
G_{X}\left(\xi^{*}\right)^{n}(\bar{F})^{n} \Rightarrow \xi^{*} G_{X}(\bar{F})^{n} \Rightarrow \xi^{*} \bar{F} G_{X}
$$

and use that $\left(\xi^{*} \circ \bar{F}\right)^{n}=\left(\xi^{*}\right)^{n} \circ(\bar{F})^{n}$. The first natural transformation comes from Lemma 4 applied for $\xi$.

\section{B.2 Proofs for Section 4.3}

In the next Theorem we only use that the fibration $p: \mathcal{E} \rightarrow \mathcal{B}$ is a bifibration and is split. ${ }^{1}$

Theorem 2. Let $\bar{T}, \bar{F}: \mathcal{E} \rightarrow \mathcal{E}$ be liftings of $T$ and $F$. If $\bar{\lambda}: \bar{T} \bar{F} \Rightarrow$ $\bar{F} \bar{T}$ is a natural transformation sitting above $\lambda$, then $\coprod_{\alpha} \circ \bar{T}$ is $\bar{F}_{\xi \text {-compatible. }}$.

\footnotetext{
${ }^{1}$ The Beck-Chevalley condition is not required for the functors $\coprod_{f}$.
} 
Proof. We exhibit a natural transformation

$$
\coprod_{\alpha} \circ \bar{T} \circ \xi^{*} \circ \bar{F} \Rightarrow \xi^{*} \circ \bar{F} \circ \coprod_{\alpha} \circ \bar{T} .
$$

This is achieved in Figure 1 by pasting five natural transformations, obtained as follows:

(a) is the counit of the adjunction $\coprod_{\lambda_{X}} \dashv \lambda_{X}^{*}$.

(b) comes from $\bar{\lambda}$ being a lifting of $\lambda$, see Lemma 5 .

(c) comes from the bialgebra condition, the fibration being split, and the units and counits of the adjunctions $\coprod_{\alpha} \dashv \alpha^{*}, \coprod_{F \alpha} \dashv$ $(F \alpha)^{*}$, and $\coprod_{\lambda_{X}} \dashv \lambda_{X}^{*}$. See Lemma 6.

(d) arises since $\bar{T}$ is a lifting of $T$, using the universal property of the Cartesian lifting $(T \xi)^{*}$, see Lemma 2.

(e) comes from $\bar{F}$ being a lifting of $F$, combined with the unit and counit of the adjunction $\coprod_{\alpha} \dashv \alpha^{*}$, see Lemma 3 .

Lemma 5. Consider a fibration $p: \mathcal{E} \rightarrow \mathcal{B}$, two $\mathcal{B}$-endofunctors $F, T$ with corresponding liftings $\bar{T}, \bar{F}$. Assume $\lambda: T F \Rightarrow F T$ is a natural transformation and $\bar{\lambda}: \overline{T F} \Rightarrow \overline{F T}$ sits above $\lambda$. Then there exists a 2-cell as in the diagram below:

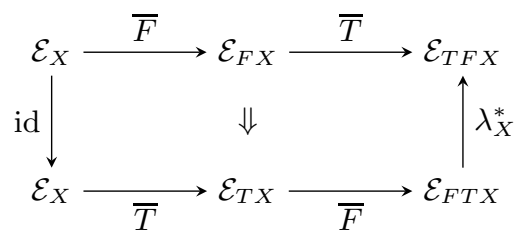

Proof. For $R \in \mathcal{E}_{F T X}$ the $R$-component of the required natural transformation is the dashed line in the diagram below and is obtained using the universal property of the Cartesian lifting of $\lambda_{X}$.

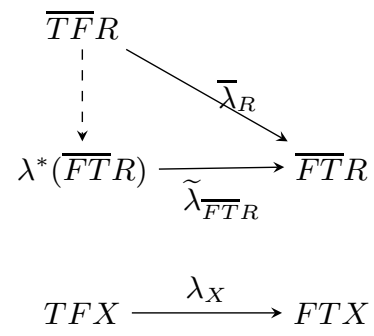

The naturality in $R$ can be easily checked and is a consequence of the uniqueness of the factorisation.

Lemma 6. Given $(X, \alpha, \xi)$ an $\lambda$-bialgebra as in (5)

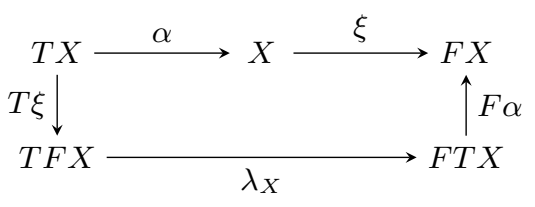

and $p: \mathcal{E} \rightarrow \mathcal{B}$ a split fibration, there exists a 2-cell

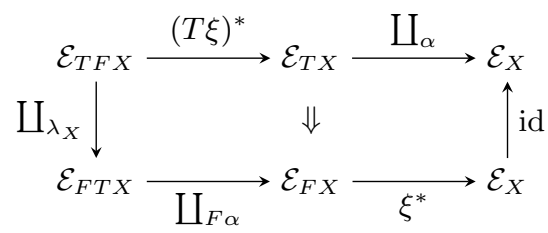

Proof. We obtain the required natural transformation as the composite of the natural transformations of (7) below.

$$
\begin{array}{cc}
\coprod_{\alpha} \circ(T \xi)^{*} & \\
\Downarrow & \left(\coprod_{\lambda} \dashv \lambda^{*}\right) \\
\coprod_{\alpha} \circ(T \xi)^{*} \circ \lambda^{*} \circ \coprod_{\lambda} & \\
\Downarrow & \left(\coprod_{F \alpha} \dashv(F \alpha)^{*}\right) \\
\coprod_{\alpha} \circ(T \xi)^{*} \circ \lambda^{*} \circ(F \alpha)^{*} \circ \coprod_{F \alpha} \circ \coprod_{\lambda} & \\
\Downarrow & (\text { bialg }) \\
\coprod_{\alpha} \circ \alpha^{*} \circ \xi^{*} \circ \coprod_{F \alpha} \circ \coprod_{\lambda} & \\
\Downarrow & \left(\coprod_{\alpha} \dashv \alpha^{*}\right) \\
\xi^{*} \circ \coprod_{F \alpha} \circ \coprod_{\lambda} &
\end{array}
$$

Except for the third one, these 2-cells are obtained from the units or counits of the adjunctions recalled on the right column. The third natural transformation is actually an isomorphism and arises from $(X, \alpha, \xi)$ being a bialgebra and the fibration being split.

\section{B.3 Proofs for Section 4.4}

In this section we will prove Lemma 1. First we recall some basic facts on the free monad $\mathrm{T}$ over a functor $T$ on some category $\mathcal{C}$.

Assuming $T$ has free algebras over any $X$ in $\mathcal{C}$ one can show that the free monad $\mathrm{T}$ over $T$ exists. We can define $\mathrm{T} X$ as the free $T$-algebra on $X$, or equivalently, as the initial algebra for the functor $X+T(-)$. Thus for each $X$ in $\mathcal{C}$ one has an isomorphism

$$
\left[\eta_{X}, \kappa_{X}\right]: X+T \mathrm{~T} X \rightarrow \mathrm{T} X .
$$

The $\eta$ above gives the unit of the monad T. The monad multiplication $\mu: \operatorname{T~T~} X \rightarrow \mathrm{T} X$ is given as the unique morphism obtained by equipping $\mathrm{T} X$ with the $\mathrm{T} X+T(-)$-algebra structure [id, $\left.\kappa_{X}\right]$.

Recall from [31] that there exists a bijective correspondence between natural transformations

$$
\lambda: T(F \times \mathrm{Id}) \Rightarrow F \mathrm{~T}
$$

and distributive laws

$$
\lambda^{\dagger}: \mathrm{T}(F \times \mathrm{Id}) \Rightarrow(F \times \mathrm{Id}) \mathrm{T} .
$$

We briefly recall here how $\lambda^{\dagger}$ is obtained from $\lambda$. For $X$ in $\mathcal{B}$, we equip $(F \times \mathrm{Id}) \mathrm{T} X$ with a $F X \times X+T(-)$-algebra structure, given by the sum of :

$$
F X \times X \longrightarrow(F \times \mathrm{Id}) \eta_{X} \longrightarrow(F \times \mathrm{Id}) \mathrm{T} X
$$

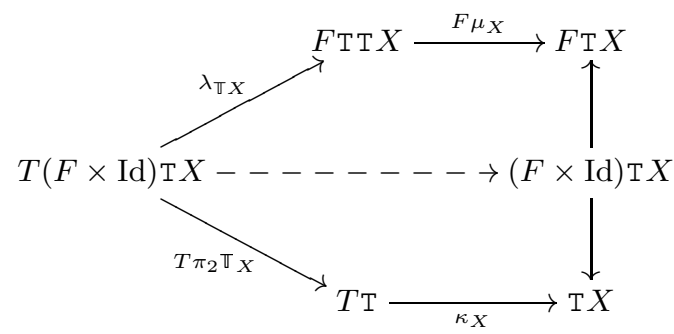


Hence $\lambda_{X}^{\dagger}$ is defined as the unique $(F \times \operatorname{Id}) X+T(-)$-algebra morphism:

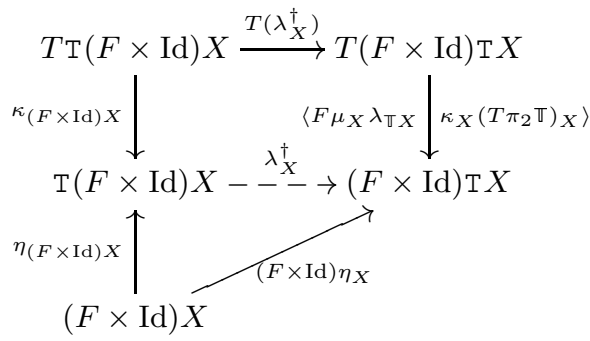

The following technical lemma is needed to establish that whenever the lifting of $\bar{T}$ of a functor $T$ has free algebras, the free monad over $\bar{T}$ is the lifting of the free monad over $T$.

Lemma 7. Consider a lifting $\bar{T}$ of a $\mathcal{B}$-endofunctor $T$ and assume $\bar{T}$ has free algebras.

1. The functor $p: \mathcal{E} \rightarrow \mathcal{B}$ has a right adjoint $\mathbf{1}: \mathcal{B} \rightarrow \mathcal{E}$ inducing an adjunction ${ }^{2}$

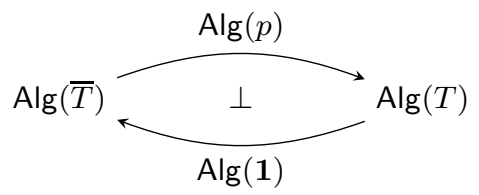

2. The functor $\operatorname{Alg}(p)$ preserves the initial algebras.

3. When $P \in \mathcal{E}_{X}$ for some $X$ in $\mathcal{B}$, the free $\bar{T}$-algebra over $P$ sits above the free $T$-algebras over $X$.

4. The free monad $\overline{\mathrm{T}}$ over $\bar{T}$ exists and is a lifting of the free monad $\mathrm{T}$ over $T$.

Proof. 1. Since the fibration considered here is bicartesian, one can define $\mathbf{1}(X)$ as the terminal object in $\mathcal{E}_{X}$. Then the statement of this item is an immediate consequence of [14, Theorem 2.14].

2. follows because $\operatorname{Alg}(p)$ is a left adjoint.

3. follows from item 1) applied for the lifting $P+\bar{T}$ of $X+T$.

4. is an immediate consequence of item 3 ).

Lemma 1. Consider a lifting $\bar{T}$ of a $\mathcal{B}$-endofunctor $T$ and assume $\bar{T}$ has free algebras. Let $\bar{\lambda}: \bar{T}(\bar{F} \times \overline{\mathrm{Id}}) \Rightarrow \overline{F \mathrm{~T}}$ be a natural transformation sitting above $\lambda: T(F \times \mathrm{Id}) \Rightarrow F \mathrm{~T}$. Then $\bar{\lambda}^{\dagger}: \overline{\mathrm{T}}(\bar{F} \times \overline{\mathrm{Id}}) \Rightarrow(\bar{F} \times \overline{\mathrm{Id}}) \overline{\mathrm{T}}$ sits above $\lambda^{\dagger}: \mathrm{T}(F \times \mathrm{Id}) \Rightarrow$ $(F \times \mathrm{Id}) \mathrm{T}$.

Proof. We know that $\mathrm{T} X$ is the free $T$-algebra on $X$. Let

$$
\left[\eta_{X}, \kappa_{X}\right]: X+T \mathrm{~T} X \rightarrow \mathrm{T} X
$$

denote the initial $X+T(-)$-algebra. Similarly, let

$$
\left[\bar{\eta}_{P}, \bar{\kappa}_{P}\right]: P+\overline{T \mathrm{~T}} P \rightarrow \overline{\mathrm{T}} P
$$

denote the initial $P+\bar{T}(-)$-algebra. By Lemma 7 we know that when $P \in \mathcal{E}_{X}$ we have that $\left[\bar{\eta}_{P}, \bar{\kappa}_{P}\right]$ is a lifting of $\left[\eta_{X}, \kappa_{X}\right]$.

\footnotetext{
${ }^{2}$ The functor Alg stems from the 2-categorical notion of inserter, see [33] or [14, Theorem 2.14,Appendix A.5] for a concise exposition.
}

For $P \in \mathcal{E}_{X}$ the map $\bar{\lambda}_{P}^{\dagger}$ is defined similarly to (8), as the unique map such that:

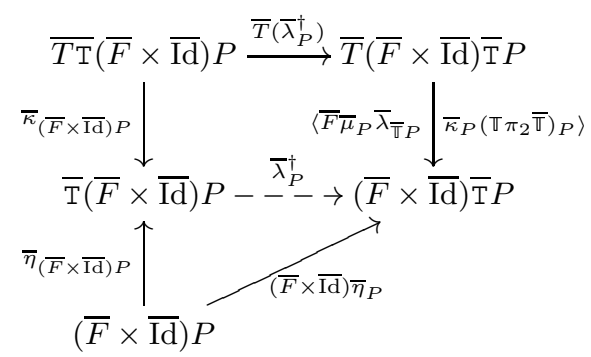

By Lemma 7 we have that the $(\bar{F} \times \overline{\mathrm{Id}}) P+\bar{T}(-)$-algebras $\overline{\mathrm{T}}(\bar{F} \times$ $\overline{\mathrm{Id}}) P$ and $(\bar{F} \times \overline{\mathrm{Id}}) \overline{\mathrm{T}} P$ of diagram (9) sit above the $(F \times \mathrm{Id}) X+$ $T(-)$-algebras $\mathrm{T}(F \times \mathrm{Id}) X$, respectively $(F \times \mathrm{Id}) \mathrm{T} X$ of diagram (8). By uniqueness of $\lambda_{X}^{\dagger}$ it follows that $\bar{\lambda}_{P}^{\dagger}$ sits above $\lambda_{X}^{\dagger}$

\section{Details on Divergence}

In this appendix, we discuss some details for showing compatibility of $C t x^{\ell}$ that were omitted in the main text for lack of space.

First of all, observe that the GSOS rules defining the parallel operator corresponds to a distributive law $\lambda: T(F \times \mathrm{Id}) \Rightarrow F \mathrm{~T}$, which is defined for all sets $X, x, y \in X$ and $S, T \in \mathcal{P}_{\omega}(L \times X)$ as

$$
\begin{aligned}
(S, x),(T, y) & \mapsto\left\{\left(l, x^{\prime} \mid y\right) \mid\left(l, x^{\prime}\right) \in S\right\} \\
& \cup\left\{\left(l, x \mid y^{\prime}\right) \mid\left(l, y^{\prime}\right) \in T\right\} \\
& \cup\left\{\left(\tau, x^{\prime} \mid y^{\prime}\right) \mid \exists a,\left(a, x^{\prime}\right) \in S \wedge\left(\bar{a}, y^{\prime}\right) \in T\right\} \\
& \cup\left\{\left(\tau, x^{\prime} \mid y^{\prime}\right) \mid \exists a,\left(\bar{a}, x^{\prime}\right) \in S \wedge\left(a, y^{\prime}\right) \in T\right\} .
\end{aligned}
$$

Intuitively, $S$ and $T$ are the sets of transitions of the states $x$ and $y$. The first set $\left\{\left(l, x^{\prime} \mid y\right) \mid\left(l, x^{\prime}\right) \in S\right\}$ corresponds to the first GSOS rule

$$
\frac{x \stackrel{l}{\rightarrow} x^{\prime}}{x\left|y \stackrel{l}{\rightarrow} x^{\prime}\right| y}
$$

and similarly for the others.

By virtue of Lemma 1, to prove compatibility of $C t x^{\ell}$, we only have to show that for all predicates $P \subseteq X$, the restriction of $\lambda_{X}$ to $\bar{T}\left(\bar{F}^{\langle\tau\rangle} \times \mathrm{Id}\right) P$ corestricts to $\bar{F}^{\langle\tau\rangle} \overline{\mathrm{T}} P$, that is whenever $(S, x),(T, y) \in \bar{T}\left(\bar{F}^{\langle\tau\rangle} \times \operatorname{Id}\right) P$, then $\lambda_{X}((S, x),(T, y)) \in$ $\bar{F}^{\langle\tau\rangle} \overline{\mathrm{T}} P$.

The latter means, by definition of $\bar{F}^{\langle\tau\rangle}$, that there exists a $(\tau, t) \in \lambda_{X}((S, x),(T, y))$ such that $t \in \overline{\mathrm{T}} P$. This can be proved as follows: since $S \in \bar{F}^{\langle\tau\rangle} P$, then there exists $\left(\tau, x^{\prime}\right) \in S$ such that $x^{\prime} \in P$. By definition of $\lambda_{X},\left(\tau, x^{\prime} \mid y\right) \in \lambda_{X}((S, x),(T, y))$. Finally, since $x^{\prime} \in P$, then $x^{\prime} \mid y \in \overline{\mathrm{T}} P$.

\section{Details on Nominal Automata}

In this section we assume the reader has some familiarity with nominal sets, see [23].

\section{D.1 The base category}

We denote by $A$ a countable set of names. The category Nom of nominal sets has as objects sets $X$ equipped with an action : : $\operatorname{Sym}(\mathrm{A}) \times X \rightarrow X$ of the group of finitely supported permutations on $A$ (that is, permutations generated by transpositions of the form $(a b))$ and such that each $x \in X$ has a finite support. Morphisms 
in Nom are equivariant functions, i.e., functions that preserve the group action.

\section{D.2 The fibration at issue}

It is well known that Nom can equivalently be described as a Grothendieck topos. Since Nom is a regular category, by [16, Observation 4.4.1] we know that the subobject fibration on Nom is in fact a bifibration. Furthermore, by a change-of-base situation described below we obtain the bifibration $\operatorname{Rel}(\mathrm{Nom}) \rightarrow$ Nom, see also [16, Example 9.2.5(ii)]

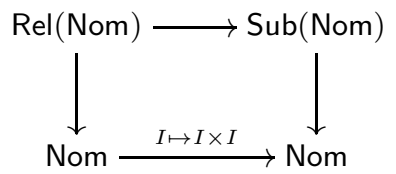

Objects of $\operatorname{Rel}(\mathrm{Nom})$ are equivariant relations. That is, if $X$ is a nominal set, a nominal relation on $X$ is just a subset $R \subseteq X^{2}$ such that $x R y$ implies $(\pi \cdot x) R(\pi \cdot y)$ for all permutations $\pi$. This bifibration is also split and bicartesian.

\section{D.3 The functors and the distributive law}

We will use the following Nom-endofunctors:

1. $F:$ Nom $\rightarrow$ Nom given by $F X=2 \times X^{\mathbb{A}}$, where $2=\{0,1\}$ is equipped with the trivial action and $X^{\mathbb{A}}$ is given by the internal hom. Concretely, an element $f \in X^{\mathbb{A}}$ is a function $f: \mathrm{A} \rightarrow X$ such that there exists a finite subset $S \subseteq \mathrm{A}$ and $f(\pi(a))=\pi \cdot f(a)$ for all names $a \in \mathrm{A}$ and permutations $\pi \in \operatorname{Sym}(\mathrm{A})$ fixing the elements of $S$.

2. $\mathcal{P}_{\omega}:$ Nom $\rightarrow$ Nom that maps a nominal set $X$ to its orbit-finite finitely supported subsets. In particular one can check that $\mathcal{P}_{\omega}$ is a monad and let $\mu$ denote its multiplication, given by union.

The functors $\mathcal{P}_{\omega}$ and $F$ are related by a distributive law

$$
\lambda: \mathcal{P}_{\omega} F \Rightarrow F \mathcal{P}_{\omega} .
$$

For a nominal set $X$, the map $\lambda_{X}$ is given by the product of the morphisms acting on $S \in \mathcal{P}_{\omega} F(X)$ by

$$
S \mapsto 1 \in 2 \text { iff } 1 \in\left(\mathcal{P}_{\omega} \tau_{1}\right)(S)
$$

and

$$
S \mapsto \lambda a .\left\{x \in X \mid \exists f \in\left(\mathcal{P}_{\omega} \tau_{2}\right)(S) . f(a)=x\right\} \in\left(\mathcal{P}_{\omega} X\right)^{A}
$$

where $\tau_{1}, \tau_{2}$ are the projections from $F X$ to 2 , respectively $X^{\mathbb{A}}$.

\section{D.4 The liftings}

The distributive law $\lambda$ can be lifted to $\operatorname{Rel}($ Nom), see [17, Exercise 4.4.6].

$$
\operatorname{Rel}(\lambda): \operatorname{Rel}\left(\mathcal{P}_{\omega}\right) \operatorname{Rel}(F) \Rightarrow \operatorname{Rel}(F) \operatorname{Rel}\left(\mathcal{P}_{\omega}\right) .
$$

Concretely, for $R \in \operatorname{Rel}(\operatorname{Nom})_{X}$, the nominal relation $\operatorname{Rel}(F)(R)$ is given by $(o, f) \operatorname{Rel}(F)(R)\left(o^{\prime}, f^{\prime}\right)$ iff $o=o^{\prime}$ and for all $a \in \AA$ we have $f(a) R f^{\prime}(a)$.

On the other hand $\operatorname{Rel}\left(\mathcal{P}_{\omega}\right)$ is given by $S \operatorname{Rel}\left(\mathcal{P}_{\omega}\right)(R) S^{\prime}$ iff for all $x \in S$ exists $y \in S^{\prime}$ with $x R y$ and for all $y \in S^{\prime}$ exists $x \in S$ with $x R y$. As for $\operatorname{Rel}(\lambda)_{R}$, this is obtained as the restriction of $\lambda_{R} \times \lambda_{R}$ to $\operatorname{Rel}\left(\mathcal{P}_{\omega}\right) \operatorname{Rel}(F)(R)$.

\section{D.5 Soundness of bisimulation up to congruence}

Nondeterministic nominal automata [4] can be modelled as $F \mathcal{P}_{\omega}$ coalgebras, while deterministic nominal automata are represented as $F$-coalgebras. The classical notion of finiteness is replaced by orbit-finiteness-from a categorical perspective this makes sense, since orbit-finite nominal sets are exactly the finitely presentable objects in the lfp category Nom.

The generalised powerset construction [28] can be applied in this situation as well, that is, a nondeterministic nominal automata modelled as a coalgebra

$$
\langle o, t\rangle: X \rightarrow 2 \times \mathcal{P}_{\omega}(X)^{A}
$$

yields an $F$-coalgebra structure

$$
\left\langle o^{\sharp}, t^{\sharp}\right\rangle: \mathcal{P}_{\omega} X \rightarrow 2 \times\left(\mathcal{P}_{\omega} X\right)^{A},
$$

on $\mathcal{P}_{\omega} X$, given by the composite $F(\mu) \circ \lambda \circ \mathcal{P}_{\omega}(\langle o, t\rangle)$. The reason why determinisation fails in a nominal setting [4] is that the finitary power object functor $\mathcal{P}_{\omega}$ does not preserve orbit finiteness. This is the case in the example of Section 5.3.

Notice that $\left(\mathcal{P}_{\omega} X, \mu,\left\langle o^{\sharp}, t^{\sharp}\right\rangle\right)$ is a $\lambda$-bialgebra.

The fibrations $\operatorname{Rel}(\mathrm{Nom}) \rightarrow$ Nom and $\mathrm{Sub}(\mathrm{Nom}) \rightarrow$ Nom are well-founded in the sense of [13]. To prove this we can apply [13, Lemma 3.4], which gives as a sufficient condition for wellfoundedness: that the fibre above each finitely presentable object be finite. Indeed, recall from [32] that finitely presentable nominal sets are the orbit-finite ones. Then, it is easy to check that a nominal set with $n$ orbits has $2^{n}$ equivariant nominal subsets.

Hence, by [Theorem 3.7][13], the final $\operatorname{Rel}(F)_{\langle o, t\rangle}$-coalgebra exists and can be computed as the limit of an $\omega^{o p}$-chain in the fibre $\operatorname{Rel}(\operatorname{Nom})_{X}$. We will use this coinductive predicate to prove that two states of a nominal automata accept the same language.

We can apply Theorem 2 to prove that the contextual closure $C t x=\coprod_{\mu} \circ \operatorname{Rel}\left(\mathcal{P}_{\omega}\right)$ is $\operatorname{Rel}(F)_{\langle o \sharp, t \sharp\rangle}$-compatible.

Thus bisimulation up to context is a valid proof technique for nominal automata.

Moreover, we can apply Proposition 2 to prove compatibility of the up to reflexive, symmetric and transitive closure techniques, respectively.

$(n=0)$ Let $R f l:$ Nom $\rightarrow \operatorname{Rel}($ Nom $)$ be the functor mapping each nominal set $X$ to $\Delta_{X}$, the identity relation on $X$. Then $R f l_{X}$ is $\operatorname{Rel}(F)_{\langle o, t\rangle}$-compatible since $\Delta_{F X}=\operatorname{Rel}(F) \Delta_{X}$.

$(n=1)$ Let $S y m: \operatorname{Rel}(\operatorname{Nom}) \rightarrow \operatorname{Rel}($ Nom $)$ be the functor mapping each nominal relation $R \subseteq X^{2}$ to its converse $R^{-1} \subseteq X^{2}$. Sym $_{X}$ is $\bar{F}_{\langle o, t\rangle}$-compatible since $\bar{F}(R)^{-1} \subseteq \bar{F}\left(R^{-1}\right)$ for all relations $R \subseteq X^{2}$.

$(n=2)$ Let $\otimes: \operatorname{Rel}(\operatorname{Nom}) \times_{\text {Nom }} \operatorname{Rel}(\operatorname{Nom}) \rightarrow \operatorname{Rel}($ Nom $)$ be the nominal relational composition functor. Composition of nominal relations is computed just as in Set and one can show that $\operatorname{Rel}(F)$ preserves it. Thus $\otimes$ is $\operatorname{Rel}(F)_{\langle o, t\rangle}$-compatible.

Employing Proposition 1 and the fact that congruence closure is obtained as the composition of the equivalence, context and reflexive closure functors we derive that bisimulation up to congruence is a sound technique.

\section{D.6 The concrete example}

The nondeterministic nominal automaton of Section 5.3 (reported on the left below) is given formally by an $F \mathcal{P}_{\omega}$-coalgebra $\langle o, t\rangle$ on the nominal set $1+1+\mathrm{A}+\mathrm{A}+1$. For simplicity we denote the 

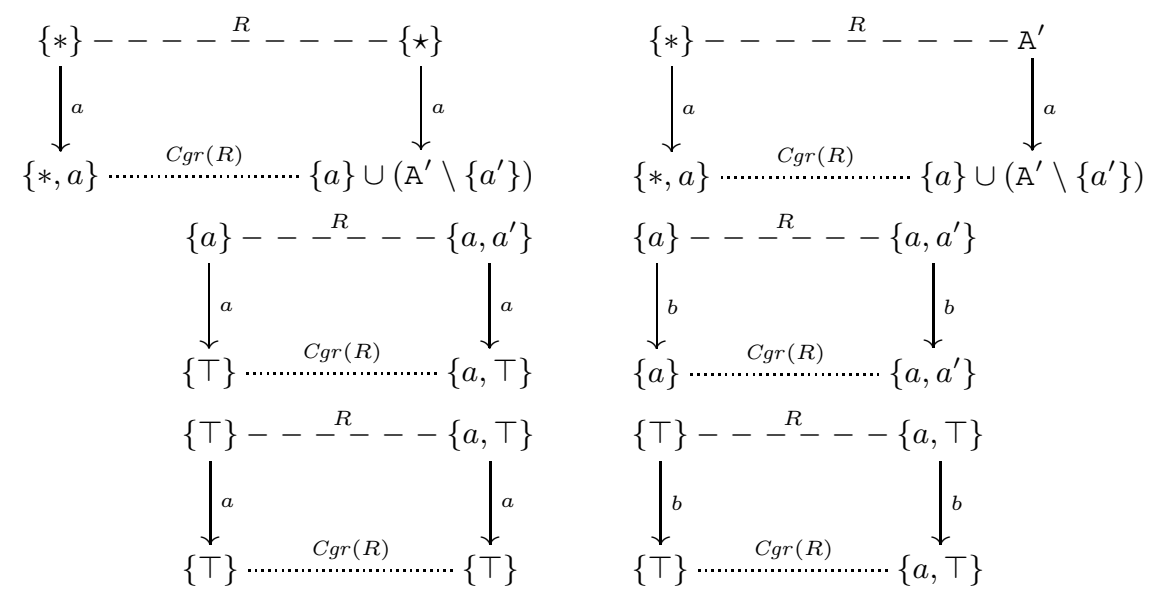

Figure 2. Proving $R$ to be a bisimulation up to congruence

second copy of $\mathrm{A}$ by $\mathrm{A}^{\prime}$. The map $\langle o, t\rangle$ is given below on the right.

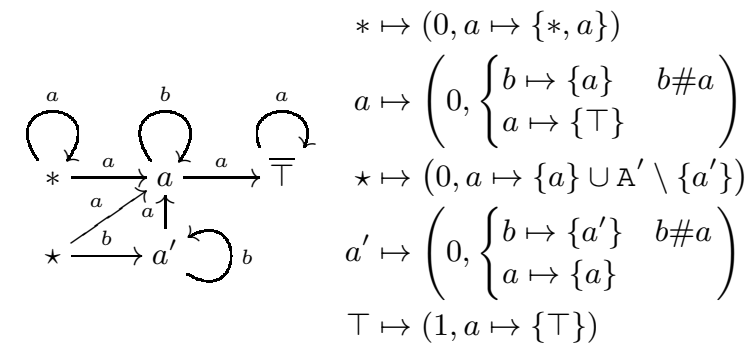

The determinisation of this automaton has infinitely many orbits. For example, the determinisation of the part reachable from $*$ is partially represented by

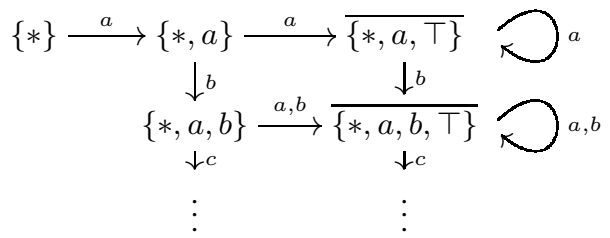

However, we can prove that $*$ and $\star$ accept the same language, showing that the nominal relation $R$ spanned by

$$
(\{*\},\{\star\}),\left(\{a\},\left\{a, a^{\prime}\right\}\right),(\{\top\},\{a, \top\}),\left(\{*\}, \mathrm{A}^{\prime}\right)
$$

is a bisimulation up to congruence, that is, $R \subseteq \operatorname{Rel}(F)_{\left\langle o^{\sharp}, t^{\sharp}\right\rangle} \operatorname{Cgr}(R)$.

This is shown in Figure 2: for each pair in $R$, we check that the successors are in $\operatorname{Cgr}(R)$. Note that for the pairs $\left(\{a\},\left\{a, a^{\prime}\right\}\right)$ and $(\{\top\},\{a, \top\})$, in the second and third rows, one needs to check the successors for $a$ and for a fresh name $b$. Instead for the pairs $(\{*\},\{\star\})$ and $\left(\{*\}, \mathrm{A}^{\prime}\right)$ in the first row, only successors for $a$ should be checked (since $a$ does not belong to the support of these states).

The only non-trivial computation is to check whether $\{*, a\} C g r(R)\{a$ $\left(\mathrm{A}^{\prime} \backslash\left\{a^{\prime}\right\}\right)$. We proceed as follows:

$$
\begin{array}{lll}
\{*, a\} & C g r(R) & \{a\} \cup \mathrm{A}^{\prime} \\
& C g r(R) & \left\{a, a^{\prime}\right\} \cup\left(\mathrm{A}^{\prime} \backslash\left\{a^{\prime}\right\}\right) \\
& C g r(R) & \{a\} \cup\left(\mathrm{A}^{\prime} \backslash\left\{a^{\prime}\right\}\right) .
\end{array}
$$

\section{E. Proofs for Section 6}

Theorem 3. Let $\bar{T}$ be a lifting of $T$ having a $\gamma: \bar{T} \otimes \Rightarrow \otimes \bar{T}^{2}$ above Id: $T \Rightarrow T$. Let both $\overline{F_{1}}$ and $\overline{F_{2}}$ be liftings of $F$. If $\lambda_{1}: \bar{T} \overline{F_{1}} \Rightarrow \overline{F_{1}} \bar{T}$ and $\lambda_{2}: \bar{T} \overline{F_{2}} \Rightarrow \overline{F_{2}} \bar{T}$ sit above the same $\lambda: T F \Rightarrow F T$, then there exists $\bar{\lambda}: \bar{T}\left(\overline{F_{1}} \otimes \overline{F_{2}}\right) \Rightarrow\left(\overline{F_{1}} \otimes \overline{F_{2}}\right) \bar{T}$ above $\lambda$.

Proof. Since $\overline{F_{1}}$ and $\overline{F_{2}}$ are liftings of $F: \mathcal{B} \rightarrow \mathcal{B}$ it follows that $\left\langle\overline{F_{1}}, \overline{F_{2}}\right\rangle: \mathcal{E} \rightarrow \mathcal{E} \times_{\mathcal{B}} \mathcal{E}$ is a lifting of $F$. Moreover $\left\langle\lambda_{1}, \lambda_{2}\right\rangle:$ $\bar{T}^{2}\left\langle\overline{F_{1}}, \overline{F_{2}}\right\rangle \Rightarrow\left\langle\overline{F_{1}}, \overline{F_{2}}\right\rangle \bar{T}$ is a lifting of $\lambda$.

Using that $\otimes: \mathcal{E} \times_{\mathcal{B}} \mathcal{E} \rightarrow \mathcal{E}$ lifts the identity we get that $F_{1} \otimes F_{2}=\otimes \circ\left\langle F_{1}, F_{2}\right\rangle$ is also a lifting of $F$.

$$
\begin{gathered}
\bar{T} \otimes\left\langle F_{1}, F_{2}\right\rangle \stackrel{\gamma\left\langle F_{1}, F_{2}\right\rangle}{\Longrightarrow} \otimes \bar{T}^{2}\left\langle F_{1}, F_{2}\right\rangle \stackrel{\otimes\left\langle\lambda_{1}, \lambda_{2}\right\rangle}{\Longrightarrow} \otimes\left\langle F_{1}, F_{2}\right\rangle \bar{T} \\
T F=T F=\frac{\lambda}{\Longrightarrow} T T
\end{gathered}
$$

The required $\bar{\lambda}$ is obtained as the composite $\otimes\left\langle\lambda_{1}, \lambda_{2}\right\rangle \circ \gamma\left\langle F_{1}, F_{2}\right\rangle$ sitting above $\lambda$ as in (10).

\section{E.1 Proofs for Similarity}

Proposition 4. Let $\lambda$ be a monotone abstract GSOS specification and $(X, \alpha,\langle\xi, i d\rangle)$ be a $\lambda^{\dagger}$-bialgebra. Then $\operatorname{Ctx}$ is $(\operatorname{Rel}(F) \sqsubseteq \times$ $\mathrm{Id})_{\langle\xi, i d\rangle}$-compatible.

Proof. Recall that Ctx is defined as $\coprod_{\alpha} \circ \operatorname{Rel}(\mathrm{T})$ and that, for the canonical lifting, it holds that $\operatorname{Rel}(\mathrm{T}) \otimes \subseteq \otimes \operatorname{Rel}(\mathrm{T})^{2}$. We decompose the lifting $\operatorname{Rel}(F) \sqsubseteq \times \operatorname{Id}$ as

$$
(\bar{\Xi} \times \overline{\mathrm{Id}}) \otimes(\operatorname{Rel}(F) \times \mathrm{Id}) \otimes(\bar{\Xi} \times \overline{\mathrm{Id}})
$$

where $\overline{\mathrm{Id}}$ is the constant functor mapping $R \subseteq X^{2}$ to $\Delta_{X}$. By Theorem 3 we reduce the proof of the fact that $\operatorname{Rel}(T)$ distributes over $\operatorname{Rel}(F) \sqsubseteq \times$ Id to the fact that $\operatorname{Rel}(T)$ distributes over $\sqsubseteq \times \overline{\mathrm{Id}}$ and $\operatorname{Rel}(F) \times$ Id separately.

For the latter, observe that $\operatorname{Rel}(F) \times \operatorname{Id}=\operatorname{Rel}(F \times \operatorname{Id})$. Since $\operatorname{Rel}(-)$ is a 2-functor [17, Exercise 4.4.6], we take $\overline{\lambda_{1}^{\dagger}}: \operatorname{Rel}(\mathrm{T}) \operatorname{Rel}(F \times$ $\mathrm{Id}) \Rightarrow \operatorname{Rel}(F \times \operatorname{Id}) \operatorname{Rel}(T)$ as $\operatorname{Rel}(\lambda)$.

For the former we need to use Lemma 1 and exhibit a $\bar{\lambda}: \operatorname{Rel}(T)(\bar{\Xi} \times$ $\overline{\mathrm{Id}}) \Rightarrow \overline{\mathrm{Rel}}(\mathrm{T})$ sitting above $\lambda$. This amounts to show that, for all relations $R \subseteq X^{2}$, the restriction of $\lambda_{X} \times \lambda_{X}$ to $\operatorname{Rel}(T)(\bar{\Xi} \times \overline{\mathrm{Id}}) R$ corestricts to $\bar{\Xi} \operatorname{Rel}(\mathrm{T}) R$. Note that since $\bar{\Xi}$ and $\overline{\mathrm{Id}}$ are constant, this is exactly the condition for monotone abstract GSOS. This guarantees the existence of $\overline{\lambda_{2}^{\dagger}}: \operatorname{Rel}(\mathrm{T})(\bar{\Xi} \times \overline{\mathrm{Id}}) \Rightarrow(\bar{\sqsubseteq} \times \overline{\mathrm{Id}}) \operatorname{Rel}(\mathrm{T})$ sitting above $\lambda^{\dagger}$. 
The existence of $\overline{\lambda_{1}^{\dagger}}$ and $\overline{\lambda_{2}^{\dagger}}$ ensures, via Theorems 3 and 2, that $C t x$ is $(\operatorname{Rel}(F) \sqsubseteq \times \operatorname{Id})_{\langle\xi, i d\rangle}$-compatible.

\section{E.2 Proofs for Weak Bisimilarity}

Lemma 8. $(\overline{F \times F}, F)$ is a fibration map.

Proof. Let $f: X \rightarrow Y$ be a function and $R \subseteq X^{2}$ be a relation. Then

$$
\begin{aligned}
& \overline{F \times F}\left(\left(f \times f^{-1}(R)\right)\right. \\
& =\{(S, U, V, W) \mid \\
& \forall(a, x) \in S . \exists(a, y) \in W \cdot f(x) R f(y), \\
& \quad \forall(a, y) \in V . \exists(a, x) \in U \cdot f(x) R f(y)\} \\
& =\{(S, U, V, W) \mid \\
& \quad \forall\left(a, x^{\prime}\right) \in F f[S] . \exists\left(a, y^{\prime}\right) \in F f[W] \cdot x^{\prime} R y^{\prime}, \\
& \left.\forall\left(a, y^{\prime}\right) \in F f[V] . \exists\left(a, x^{\prime}\right) \in F f[U] \cdot x^{\prime} R y^{\prime}\right\} \\
& =(F f \times F f \times F f \times F f)^{-1}(\overline{F \times F}(R))
\end{aligned}
$$

Proposition 5. Let $\lambda: T(F \times \mathrm{Id}) \Rightarrow F \mathrm{~T}$ be a positive GSOS specification and $\left(X, \alpha,\left\langle\xi_{1}, i d\right\rangle\right)$ and $\left(X, \alpha,\left\langle\xi_{2}, i d\right\rangle\right)$ be two $\lambda^{\dagger}$ bialgebras then Ctx is $(\overline{F \times F} \times \mathrm{Id})_{\left\langle\xi_{1}, \xi_{2}, i d\right\rangle}$-compatible.

Proof. From $\lambda: T(F \times \mathrm{Id}) \Rightarrow F \mathrm{~T}$, we define $\tilde{\lambda}: T(F \times F \times \mathrm{Id}) \Rightarrow$ $(F \times F) \mathrm{T}$ as $\left\langle\lambda \circ T\left(\tau_{1} \times \tau_{3}\right), \lambda \circ T\left(\tau_{2} \times \tau_{3}\right)\right\rangle$ where $\tau_{i}$ are the projections from $F \times F \times$ Id to $F$ and Id. Such $\tilde{\lambda}$ induces a distributive law

$$
\tilde{\lambda}^{\dagger}: \mathrm{T}(F \times F \times \mathrm{Id}) \Rightarrow(F \times F \times \mathrm{Id}) \mathrm{T} .
$$

From the $\lambda^{\dagger}$-bialgebras $\left(X, \alpha,\left\langle\xi_{1}, i d\right\rangle\right)$ and $\left(X, \alpha,\left\langle\xi_{2}, i d\right\rangle\right)$, we construct $\left(X, \alpha,\left\langle\xi_{1}, \xi_{2}, i d\right\rangle\right)$ which is a $\tilde{\lambda}^{\dagger}$-bialgebra.

Recall that $C t x$ is defined as $\coprod_{\alpha} \circ \operatorname{Rel}(\mathrm{T})$ and that, for the canonical lifting, it holds that $\operatorname{Rel}(\mathrm{T}) \otimes \subseteq \otimes \operatorname{Rel}(\mathrm{T})^{2}$. We decompose the lifting $\overline{F \times F} \times \operatorname{Id}$ as

$$
(\rho \times \overline{\mathrm{Id}}) \otimes\left(\operatorname{Rel}(F \times F)^{[\supseteq \subseteq]} \times \mathrm{Id}\right)
$$

where $\overline{\mathrm{Id}}$ is the constant functor mapping $R \subseteq X^{2}$ to $\Delta_{X}$. By Theorem 3 we reduce the proof of the fact that $\operatorname{Rel}(T)$ distributes over $\overline{F \times F} \times \operatorname{Id}$ to the fact that $\operatorname{Rel}(T)$ distributes over $\rho \times \overline{\mathrm{Id}}$ and $\operatorname{Rel}(F \times F)^{[\supseteq \subseteq]} \times$ Id separately.

For the former, by Lemma 1 , we have to prove that for all relations $R \subseteq X^{2}$, the restriction of $\tilde{\lambda}_{X} \times \tilde{\lambda}_{X}$ to $\operatorname{Rel}(T)(\rho \times \overline{\mathrm{Id}}) R$ corestricts to $\rho \operatorname{Rel}(\mathrm{T}) R$. This can be easily checked by using the fact that both $\rho$ and $\overline{\mathrm{Id}}$ are constant and exploiting the definition of $\tilde{\lambda}$. As a consequence there exists a ${\tilde{\lambda_{1}}}^{\dagger}: \operatorname{Rel}(\mathrm{T})(\rho \times \overline{\mathrm{Id}}) \Rightarrow$ $(\rho \times \overline{\mathrm{Id}}) \operatorname{Rel}(\mathrm{T})$ sitting above $\tilde{\lambda}^{\dagger}$.

For $\operatorname{Rel}(F \times F)^{[\supseteq \subseteq]} \times$ Id we can reuse Proposition 4 , but first we have to prove that the GSOS specification $\tilde{\lambda}$ is monotone w.r.t. $[\supseteq \subseteq]$. Via simple computations, one can check that this is indeed the case when the original GSOS specification $\lambda$ is positive. As a consequence there exists a ${\tilde{\lambda_{2}}}^{\dagger}: \operatorname{Rel}(\mathrm{T})\left(\operatorname{Rel}(F \times F)^{[\supseteq \subseteq]} \times \operatorname{Id}\right) \Rightarrow$ $\left(\operatorname{Rel}(F \times F)^{[\supseteq \subseteq]} \times \operatorname{Id}\right) \operatorname{Rel}(\mathrm{T})$ sitting above $\tilde{\lambda}^{\dagger}$.

The existence of ${\tilde{\lambda_{1}}}^{\dagger}$ and ${\tilde{\lambda_{2}}}^{\dagger}$ entails, via Theorems 3 and 2 compatibility of $C t x$ for $(\overline{F \times F} \times \mathrm{Id})_{\left\langle\xi_{1}, \xi_{2}, i d\right\rangle}$.

\section{Additional references for the appendix}

[32] D. Petrişan. Investigations into Algebra and Topology over Nominal Sets. PhD thesis, University of Leicester, 2012.

[33] R. Street. Fibrations and Yoneda's lemma in a 2-category. In GregoryM. Kelly, editor, Category Seminar, volume 420 of Lecture Notes in Mathematics, pages 104-133. Springer Berlin Heidelberg, 1974. 